Article

\title{
Analyst Following, Group Affiliation, and Labor Investment Efficiency: Evidence from Korea
}

\author{
Kyoungwon $\mathrm{Mo}^{1}$ and Kyung Yun (Kailey) Lee ${ }^{2, *} \mathbb{B}$ \\ 1 School of Business Administration, Chung-Ang University, Seoul 06974, Korea; kwmo@cau.ac.kr \\ 2 College of Business Administration, Hankuk University of Foreign Studies, Seoul 02450, Korea \\ * Correspondence: kylee@hufs.ac.kr; Tel.: +82-2-2173-3080
}

Received: 3 May 2019; Accepted: 31 May 2019; Published: 4 June 2019

\begin{abstract}
This paper studies how analysts' group affiliation affects firms' labor investment efficiency. Using a 2001-2017 sample of Korean public companies, we find that labor investment efficiency increases when there are more unaffiliated analysts following business group (chaebol) firms. Our regression results also suggest that an increase in labor investment efficiency is attributed to a reduction in firms' over-firing problem. However, affiliated analysts are not found to influence firms' labor investment efficiency. We further document that the positive influence of unaffiliated analysts on labor investment efficiency holds when firms have high cash holdings. Our results are robust to different model specifications, including two-stage least square regression and firm-size matching.
\end{abstract}

Keywords: analyst following; group-affiliated analysts; chaebol; labor investment efficiency

\section{Introduction}

It is well known that analysts actively communicate with the senior managers of companies and serve an important role in corporate policy decisions. In a survey of over 300 analysts, more than half answered that they have direct contact with Chief Executive Officers (CEOs) or Chief Financial Officers (CFOs) at least five times a year [1]. Analysts mentioned that conversations with senior managers are a very useful source when they make stock recommendations or earnings forecasts. On the other hand, Graham, Harvey, and Rajgopal showed that many CFOs view analysts as the most important investors in terms of setting the stock price for their companies [2]. Senior managers reported that analysts affect their decisions on corporate policies, as meeting analyst benchmarks is an important consideration for them. Therefore, analysts and corporate managers are talking to each other in the hope that they can obtain or deliver valuable information about the firm, which can affect corporate policy and thus stimulate corporate sustainability.

Researchers in prior studies have focused on the role of analysts on corporate sustainability. For example, Yu showed that managers are less likely to manage earnings when there are more analysts following [3]. Also, Chen, Harford, and Lin argued that analyst following increases cash holdings and decreases CEOs' excess compensation, value-destroying acquisitions, and earnings management [4]. In practice, analysts not only forecast companies' earnings, but they also voice their opinions on corporate policies. In July 2018, when Ford Motor Company announced restructuring plans, some analysts responded positively. One called the decision an encouraging sign for investors of the company's confidence in its ability to advance the brand [5]. Analysts may also provide opinions on how firms can survive after acquisitions. For example, analysts suggested several ways to make Occidental Petroleum's acquisition of Anadarko Petroleum successful. Specifically, analysts proposed that the success of the acquisition depends on how quickly Occidental can sell off Anadarko's assets in the Gulf of Mexico, and "the longer Occidental take to reduce debt from the deal, the longer they stay in the doghouse with investors" [6]. 
Analysts also share their thoughts on firms' employment decisions. For instance, when retail industries were cutting labor costs due to higher market competition, analysts predicted the negative effect of low staffing. They specifically said that reducing labor costs could compound sales problems over time [7]. In other cases, analysts can also support companies' layoff decisions. For example, Cisco Systems Inc. cut thousands of jobs in 2011. When they announced this plan, analysts mentioned that "they were pleased to see Cisco taking quick and decisive action on restructuring," while also mentioning, "We all love the billion dollars in cost savings, but you never cheer people losing their jobs" [8].

Labor investment as well as capital investment is one of the most important corporate decisions which determines a firm's sustainability. The effect of capital investment on firms' sustainability has been examined in prior studies. $\mathrm{Xu}$ and Sim noted that firms must allocate their resources efficiently to achieve a sustainable performance. Specifically, authors find that research and development (R\&D) investment has a positive effect on future performance for both Chinese and Korean firms [9]. Moreover, using unexpected levels of capital investment to measure investment inefficiency, Oh and Kim found that a negative association between seasoned equity offering (SEO) and investment efficiency disappears when there are more active analysts. This result emphasizes the importance of analysts on corporate sustainability [10].

Based on analysts' influence on firms' employment policies, in this study, we investigate whether analysts' governance role affects companies' labor investment efficiency. Specifically, the purpose of this study is to examine whether analysts' group affiliation affects the labor investment efficiency of the companies they follow. There exist two competing theories on whether affiliated analysts will increase labor investment efficiency: information sharing theory and conflict-of-interest theory. If an analyst is affiliated with a particular firm, he or she will have a higher chance of contacting senior management through conference calls and thus be able to provide greater monitoring with inside information. If this is the case, compared to unaffiliated analysts, affiliated analysts are more likely to exert a higher governance role, which can increase the labor investment efficiency of companies within the group. On the other hand, analysts may have a bias towards firms in the same group affiliation. If they do, rather than providing a monitoring effect, they may produce biased earnings forecasts and form public opinion as opinion leaders (conflict-of-interest theory). For instance, Huyghebaert and $\mathrm{Xu}$ showed that affiliated analysts issue more positively biased forecasts, target prices, and investment recommendations compared to independent analysts [11]. They noted that affiliated analysts might be genuinely more optimistic about the firms they follow, or they might strategically issue their reports due to improper incentives. These two conflicting theories indicate that whether affiliated analysts positively affect labor investment efficiency is an empirical question.

We group analysts depending on their affiliation with business groups (chaebols). In Korea, the top ten chaebols own more than 27 percent of all business assets [12]. Given the importance of chaebols in the economy, Korea provides a unique setting where non-financial companies can own financial-securities or insurance-firms, as long as the debt-to-equity ratio of the group is below 200 percent. Prior studies have examined the quality of earnings forecasts and stock recommendations by analysts who work for financial firms that belong to one of the chaebols [13]. Although the virtues of analysts are generally independence and fairness, the situation in Korea is a bit different. In April 2016, the heads of 32 local securities firms gathered to create a joint statement to oppose firms that pressured analysts not to release unfavorable reports about them [14]. These firms often pressure analysts to report earnings estimates optimistically by threatening not to give private information to the analysts. By understanding the different institutional environment of analysts, we believe that examining whether analysts' group affiliation affects firms' labor investment efficiency in the Korean setting will shed light on the factors that influence corporate policy choices. 
Overall, we find that analysts' affiliation with chaebols affects labor efficiency, supporting the conflict-of-interest hypothesis. Specifically, using data on Korean public companies from 2001 to 2017, we test the effect of analyst following on abnormal net hiring and show that there is a positive association between non-affiliated analysts and labor efficiency in chaebol firms. Further tests indicate that an increase in labor efficiency comes from preventing companies from firing too many of their employees. We find that this positive effect of non-affiliated analysts on labor efficiency becomes stronger when there is more inside funding. Since analyst following is associated with many factors, a two-stage least squares regression (2SLS) model is used to mitigate endogeneity concerns.

While previous studies have shown analysts' positive influence on firms, this study adds to the literature by showing that not all analysts are the same: they may have different influence on firms they follow depending on whether they are independent of the firms. Specifically, we show that analysts' governance role positively affects firms' labor investment efficiency only when analysts are unaffiliated. We test this relation using Korean public firms, as Korea provides a unique setting where non-financial firms can own financial firms, and analysts from these financial firms provide reports on firms in their group (chaebol). Several papers have discussed the lower quality of information produced by group-affiliated analysts via their earnings forecasts and stock recommendations $[13,15,16]$. Yet, to the best of our knowledge, there is no research regarding analysts' influence on corporate decisions, other than their role in the stock market. This paper contributes to the literature by confirming that the conflict-of-interest theory also applies to the relationship between analysts' group affiliation and the labor market. In other words, the findings in this paper suggest that affiliated analyst following tends to preclude firms from having a sustainable employment policy. Thus, unaffiliated analysts are likely to improve firms' labor investment decisions, which can further lead to an increase in firms' sustainability.

Section 2 outlines the research design, including explanations of the definitions of the key variables. Section 3 presents the empirical results on the relationship between analysts' group affiliation and labor investment efficiency. Section 4 discusses additional tests, and Section 5 outlines the conclusions of the paper.

\section{Key Variable Definition and Research Design}

\subsection{Specification of Labor Investment Efficiency}

We measure labor investment inefficiency using abnormal net hiring, which is defined as subtracting expected net hiring from actual net hiring. According to prior studies, the expected change in labor for firm $i$ in year $t$ is estimated using the following regression model with the firms' economic fundamentals $[17,18]$ :

$$
\begin{aligned}
& N E T \_H I R E_{i, t}=\alpha_{0}+\alpha_{1} S A L E S \_G R O W T H_{i, t-1}+\alpha_{2} S A L E S \_G R O W T H_{i, t}+\alpha_{3} \Delta R O A_{i, t-1} \\
& +\alpha_{4} \Delta R O A_{i, t}+\alpha_{5} R O A_{i, t}+\alpha_{6} R_{E T U R N}{ }_{i, t}+\alpha_{7} S I Z E_{-} R_{i, t-1}+\alpha_{8} \text { Quick }_{i, t-1} \\
& +\alpha_{9} \Delta \text { Quick }_{i, t-1}+\alpha_{10} \Delta \text { Quick }_{i, t}+\alpha_{11} L_{E V_{i, t-1}}+\alpha_{12} \text { LOSSBIN1 }_{i, t-1} \\
& +\alpha_{13} \text { LOSSBIN }_{i, t-1}+\alpha_{14} \text { LOSSBIN }_{i, t-1}+\alpha_{15} \text { LOSSBIN }_{i, t-1} \\
& +\alpha_{16} L_{O S S B I N 5} 5_{i, t-1}+\text { Industry Fixed Effects }+\varepsilon_{i, t}
\end{aligned}
$$

where NET_HIRE $E_{i, t}$ is the percentage change in number of employees at year $t$; SALES_GROWTH $H_{i, t-1}\left(S A L E S \_G R O W T H_{i, t}\right)$ is the percentage change in sales revenue at year $t-1$ (year $t) ; R O A_{i, t}$ is return on assets, which is net income at year $t$ scaled by total assets at year $t-1$; $\triangle R O A_{i, t-1}\left(\triangle R O A_{i, t}\right)$ is the change in ROA at year $t-1$ (year $\left.t\right) ; R E T U R N_{i, t}$ is the total annual stock return for year $t$; SIZE_ $R_{i, t-1}$ is the natural logarithm of the market value of equity at the beginning of the year, ranked into percentiles; Quick ${ }_{i, t-1}$ is the quick ratio at year $t-1$, which is calculated by the sum of cash and cash equivalents, short-term investments, and receivables, divided by current liabilities; $\Delta$ Quick $_{i, t-1}\left(\Delta\right.$ Quick $\left._{i, t}\right)$ is the change in the quick ratio at year $t-1$ (year $\left.t\right) ; L E V_{i, t-1}$ is the ratio of long-term liabilities to total assets at year $t-1$; and five $\operatorname{LOSSBIN}_{i, t-1}$ variables are used as 
an indicator of whether a firm's ROA is classified into the five small loss bins with an interval of 0.005 at year $t-1$ : $\operatorname{LOSSBIN1} 1_{i, t-1}$ equals one if a firm's ROA at year $t-1$ is between -0.005 and 0 , LOSSBIN2 $2_{i, t-1}$ equals one if a firm's ROA at year $t-1$ is between -0.01 and $-0.005, \operatorname{LOSSBIN}_{i, t-1}$ equals one if a firm's ROA at year $t-1$ is between -0.015 and $-0.01, \operatorname{LOSSBIN} 4_{i, t-1}$ equals one if a firm's ROA at year $t-1$ is between -0.02 and -0.015 , and $L O S S B I N 5_{i, t-1}$ equals one if a firm's ROA at year $t-1$ is between -0.025 and -0.02 .

If a firm has a positive (negative) abnormal net hiring, it is considered to have hired more (fewer) employees than the predicted level. For both cases, when abnormal net hiring $\left(A B \_N E T \_H I R E_{i, t}\right)$ is either high or low, firms are defined as being inefficient in labor investment. The absolute value of abnormal net hiring, $\left|A B \_N E T \_H I R E\right|_{i, t}$, is a proxy for labor investment inefficiency.

The descriptive statistics for the variables used in regression Model (1) are illustrated in Table 1, Panel A. The percentage change in employees, on average (NET_HIRE $\left.E_{i, t}\right)$, is $2.6 \%$, indicating that sample firms generally over-hire rather than over-fire employees. On average, the size of our sample firms (SIZE_R), measured by a logarithm of market value of the equity, is 0.5 . The median values of sales growth rate (SALES_GROWTH) and ROA are about $20 \%$ and $2 \%$, respectively, indicating that sample firms are profitable on average. However, both the mean and median of change in ROA is negative $(-44 \%$ and $-25 \%)$. Sample firms, on average, have a positive annual stock return (RETURN), quick ratio (Quick), and debt ratio (LEV). Table 1, Panel B presents results from regression Model (1). The model's R-square equals $8 \%$ and the F-statistics are 27.48. The coefficients of independent variables indicate that an increase in sales growth rate, the current year's performance, stock returns and size, and last year's quick ratio and change in quick ratio increase firms' employment. On the other hand, employment decreases as changes in performance, changes in quick ratio, and last year's debt ratio increase. Firms' net hiring decreases when the firm's ROA is slightly below zero, but the rest of the $\operatorname{LOSSBIN}_{i, t-1}$ variables are not statistically significant.

Table 1. Estimating the expected level of net hiring and abnormal hiring.

\begin{tabular}{|c|c|c|c|c|c|c|}
\hline \multicolumn{7}{|c|}{ Panel A: Descriptive Statistics for Variables in Model (1) } \\
\hline Variables & $\mathbf{N}$ & Mean & Median & Std. & Q1 & Q3 \\
\hline NET_HIRE $E_{i t}$ & 25,122 & 0.0261 & 0.0067 & 0.2757 & -0.0612 & 0.0860 \\
\hline SALES_GROWTH $H_{i t-1}$ & 25,122 & 0.1957 & 0.0597 & 0.7303 & -0.1242 & 0.2884 \\
\hline$S A L E \bar{S} \_G R O W T H_{i t}$ & 25,122 & 0.1734 & 0.0475 & 0.7381 & -0.1354 & 0.2603 \\
\hline$\Delta \bar{R} O A_{i t-1}$ & 25,122 & -0.4377 & -0.2573 & 4.4229 & -0.8725 & 0.2989 \\
\hline$\Delta R O A_{i t}$ & 25,122 & -0.4552 & -0.2445 & 4.9157 & -0.8843 & 0.3415 \\
\hline$R O A_{i t}$ & 25,122 & -0.0122 & 0.0253 & 0.1751 & -0.0272 & 0.0672 \\
\hline RETURN $_{i t}$ & 25,122 & 0.2158 & -0.0022 & 0.9261 & -0.2689 & 0.3737 \\
\hline$S I Z E_{-} R_{i t-1}$ & 25,122 & 0.5020 & 0.4900 & 0.2747 & 0.2700 & 0.7400 \\
\hline Quick $_{i t-1}$ & 25,122 & 1.1683 & 0.7734 & 1.6862 & 0.4571 & 1.3192 \\
\hline$\widetilde{\Delta Q u i c k}_{i t-1}$ & 25,122 & 0.1562 & 0.0002 & 0.8008 & -0.2192 & 0.2690 \\
\hline$\widetilde{\Delta Q}$ uickt $_{i t}$ & 25,122 & 0.1324 & -0.0072 & 0.7760 & -0.2232 & 0.2468 \\
\hline$L E V_{i t-1}$ & 25,122 & 0.0338 & 0.0000 & 0.0684 & 0.0000 & 0.0377 \\
\hline \multicolumn{7}{|c|}{ Panel B: Regression Results (Dependent Variable $=$ NET_HIRE) } \\
\hline \multicolumn{4}{|c|}{ Independent Variables } & Coeff. & ( $t$-Value) & \\
\hline \multicolumn{4}{|c|}{ Intercept } & 0.122 & $(1.90)$ & * \\
\hline \multicolumn{4}{|c|}{ SALES_GROWTH $H_{i t-1}$} & 0.044 & $(18.64)$ & $* * *$ \\
\hline \multicolumn{4}{|c|}{ SALES_GROWTH } & 0.078 & $(33.11)$ & $* * *$ \\
\hline \multicolumn{4}{|c|}{$\Delta R O A_{i t-1}$} & 0.000 & $(0.32)$ & \\
\hline \multicolumn{4}{|c|}{$\Delta R O A_{i t}$} & -0.001 & $(-2.00)$ & $* *$ \\
\hline \multicolumn{4}{|c|}{$R O A_{i t}$} & 0.105 & $(9.99)$ & $* * *$ \\
\hline \multicolumn{4}{|c|}{ RETURN $_{i t}$} & 0.019 & $(9.86)$ & $* * *$ \\
\hline \multicolumn{4}{|c|}{$S I Z E_{-} R_{i t-1}$} & 0.049 & $(7.20)$ & $* * *$ \\
\hline
\end{tabular}


Table 1. Cont.

\begin{tabular}{|c|c|c|c|}
\hline \multicolumn{4}{|c|}{ Panel B: Regression Results (Dependent Variable $=$ NET_HIRE) } \\
\hline Independent Variables & Coeff. & ( $t$-Value) & \\
\hline Quick $_{i t-1}$ & 0.007 & $(6.31)$ & $* * *$ \\
\hline$\widetilde{\Delta Q u i c k}_{i t-1}$ & 0.005 & $(2.16)$ & ** \\
\hline$\widetilde{\Delta Q u i c k t}_{i t}$ & -0.021 & $(-9.14)$ & $* * *$ \\
\hline$L E V_{i t-1}$ & -0.074 & $(-2.86)$ & $* * *$ \\
\hline LOSSBIN1 $_{i t-1}$ & -0.027 & $(-1.89)$ & * \\
\hline LOSSBIN2 $2_{i t-1}$ & -0.007 & $(-0.43)$ & \\
\hline $\operatorname{LOSSBIN3}_{i t-1}$ & -0.002 & $(-0.14)$ & \\
\hline LOSSBIN $4_{i t-1}$ & 0.000 & $(-0.02)$ & \\
\hline LOSSBIN5 & 0.012 & $(0.72)$ & \\
\hline Industry fixed effects & & Yes & \\
\hline [F-value] & & {$[27.48]$} & $* * *$ \\
\hline$R^{2}$ & & 0.0779 & \\
\hline $\mathrm{N}$ & & 25,122 & \\
\hline
\end{tabular}

Notes: Panel A summarizes the descriptive statistics of the variables in Model (1). Panel B reports the regression results of NET_HIRE on various control variables. T-statistics are calculated based on robust standard errors clustered at the firm level and are reported in parentheses. Statistical significance at the 1,5 , and $10 \%$ levels is denoted by ${ }^{* * *}, * *$, and $*$, respectively. See Appendix A for variable definitions.

\subsection{Specification of Analysts' Group Affiliation}

In this paper, we categorize analysts into four groups depending on whether they are from group-affiliated securities firms and whether they follow and issue forecasts for affiliated firms within the same group. Firms are defined as group-affiliated when they belong to the list of chaebols provided by the Korea Fair Trade Commission (KFTC). We follow the process of Lim and Jung (2012) to determine the number of affiliated and non-affiliated analysts following a firm. The number of affiliated analysts following within-group affiliated firms (nongroup or unaffiliated firms) is defined as GAGF (GANGF), and the number of unaffiliated analysts following group firms (nongroup firms) is defined as NGAGF (NGANGF). Using these four variables-GAGF, GANGF, NGAGF, and NGANGF-as our independent variables of interest in regression models, we aim to examine whether analysts' group affiliation affects the efficiency of firms' labor investment.

\subsection{Control Variables}

Eleven variables are used in the regression to control for other factors that might affect firms' employment decisions [19-22]. First, following Biddle and Hilary (2006), a firm's size (SIZE) and its financials, such as growth options (MTB), liquidity (Quick), and dividend payout ratio (DIVDUM), are included in the regression model. Also, we include control variables regarding a firm's financial risk: leverage (LEV), having losses (LOSS), and a tangible asset ratio (TANGIBLE). Since Liu and Wysocki showed that volatilities in the cash flows and sales revenue affect the association between accruals quality and cost of capital, we use volatility in cash flows and sales revenue (STD_CFO, STD_SALE) as control variables [21]. The model also includes institutional ownership (INSTI) to control for corporate governance, as suggested by Cella (2019) [22]. Lastly, volatility in net hiring (STD_NET_HIRE) is included in the model to ensure that our results are not simply driven by investment volatility. 
2.4. Main Regression Model

$$
\begin{aligned}
& \left|A B \_N E T_{-} H I R E\right|_{i, t}=\alpha_{0}+\beta_{1} G_{A G F_{i, t-1}}+\beta_{2} \text { GANGF }_{i, t-1}+\beta_{3} \text { NGAGF }_{i, t-1}+\beta_{4} N G A N G F_{i, t-1} \\
& +\gamma_{1} \text { MTB }_{i, t-1}+\gamma_{2} \text { SIZE }_{i, t-1}+\gamma_{3} \text { Quick }_{i, t-1}+\gamma_{4} L E V_{i, t-1}+\gamma_{5} \text { DIVDUM }_{i, t-1} \\
& +\gamma_{6} S T D_{-} C F O_{i, t-1}+\gamma_{7} S T D_{-} S A L E_{i, t-1}+\gamma_{8} T_{\text {TANGIBLE }}{ }_{i, t-1}+\gamma_{9} L O S S_{i, t-1} \\
& +\gamma_{10} I N S T I_{i, t-1}+\gamma_{11} S T D \_N E T \_H I R E_{i, t-1}+\text { Year Fixed Effects } \\
& + \text { Industry Fixed Effects }+\varepsilon_{i, t}
\end{aligned}
$$

where $\left|A B \_N E T \_H I R E\right|_{i, t}$ is abnormal net hiring, derived from Model (1); GAGF $F_{i, t-1}$ and $G A N G F_{i, t-1}$ are the number of affiliated analysts following within-group firms and unaffiliated firms, respectively; $N G A G F_{i, t-1}$ and $N G A N G F_{i, t-1}$ are the number of nongroup analyst following group firms and nongroup firms, respectively; $M T B_{i, t-1}$ is the market to book ratio, which is calculated by dividing the market value of equity by the book value of equity; $S I Z E_{i, t-1}$ is the natural logarithm of the market value of equity; Quick $k_{i, t-1}$ is the sum of cash and cash equivalents, short-term investments, and receivables, divided by current liabilities; $L E V_{i, t-1}$ is the ratio of long-term liabilities to the beginning balance of total assets; DIVDUM $M_{i, t-1}$ is an indicator variable that equals one if a firm pays dividends and zero otherwise; $S T D \_C F O_{i, t-1}$ is the standard deviation of the cash flows from operations over the most recent five years; $S T D_{-} S A L E_{i, t-1}$ is the standard deviation of sales revenue over the most recent five years; TANGIBLE $E_{i, t-1}$ is the ratio of long-term assets (property, plant, and equipment) to the beginning balance of total assets; $\operatorname{LOSS}_{i, t-1}$ is an indicator variable that equals one if a firm has a net loss and zero otherwise; INSTI $I_{i, t-1}$ is the number of shares owned by institutional investors scaled by the number of total outstanding shares; STD_NET_HIRE $E_{i, t-1}$ is the standard deviation of net hiring. Industry and year fixed effects are included in all regressions, and standard errors are clustered at the firm level.

\section{Empirical Results}

\subsection{Samples and Data}

The sample includes all firms whose stocks are publicly traded either on the Korea Composite Stock Price Index (KOSPI) or the Korea Securities Dealers Automated Quotations (KOSDAQ) from 2001 to 2017. The sample period starts in 2001, which is the year the KFTC started to release data regarding chaebol group affiliation. Firms in financial industries and observations with missing firm characteristics are excluded from the analyses. Three databases are used in this study: the analyst following and accounting data are from DataguidePro, the institutional ownership data is from TS2000, and the firms' group affiliation data is from the KFTC. The final sample consists of 7745 firm-year observations.

\subsection{Descriptive Statistics and Correlations}

Descriptive statistics of the variables we used in this study are presented in Table 2 . The mean and median values of $\left|A B \_N E T \_H I R E\right|_{i, t}$, the dependent variable, are 0.1147 and 0.0615 , respectively, which is comparable to those found in Jung, Lee, and Weber [17]. On average, 7-8 analysts are following a particular firm. Moreover, about 3.68 analysts are group-affiliated analysts following nongroup or unaffiliated firms, 2.29 are nongroup analysts following nongroup firms, 1.45 are nongroup analysts following group firms, and 0.05 are affiliated analysts following within-group firms. 
Table 2. Descriptive statistics for variables in the abnormal net hiring model (Model (2)).

\begin{tabular}{ccccccc}
\hline Variables & $\mathbf{N}$ & Mean & Median & Std. & Q1 & Q3 \\
\hline $\mid$ AB_NET_HIRE $\mid$ & 7745 & 0.1147 & 0.0615 & 0.1688 & 0.0276 & 0.1282 \\
Analyst coverage & 7745 & 7.4878 & 3.0000 & 8.5643 & 1.0000 & 11.0000 \\
GAGF & 7745 & 0.0513 & 0.0000 & 0.2960 & 0.0000 & 0.0000 \\
GANGF & 7745 & 3.6820 & 2.0000 & 4.5888 & 1.0000 & 5.0000 \\
NGAGF & 7745 & 1.4546 & 0.0000 & 3.8266 & 0.0000 & 0.0000 \\
NGANGF & 7745 & 2.2999 & 1.0000 & 3.3781 & 0.0000 & 3.0000 \\
MTB & 7745 & 0.0015 & 0.0011 & 0.0056 & 0.0006 & 0.0018 \\
SIZE & 7745 & 12.2492 & 11.9876 & 1.6809 & 11.0441 & 13.1880 \\
Quick & 7745 & 1.0674 & 0.7648 & 1.3079 & 0.4869 & 1.2317 \\
LEV & 7745 & 0.0386 & 0.0000 & 0.0667 & 0.0000 & 0.0602 \\
DIVDUM & 7745 & 0.7584 & 1.0000 & 0.4281 & 1.0000 & 1.0000 \\
STD_CFO & 7745 & $108,945,561$ & $13,449,562$ & $436,717,500$ & $5,350,999$ & $44,152,740$ \\
STD_SALE & 7745 & $484,220,140$ & $48,324,906$ & $2,144,862,028$ & $17,216,405$ & $171,754,769$ \\
TANGIBLE & 7745 & 0.3335 & 0.3278 & 0.1812 & 0.1991 & 0.4556 \\
LOSS & 7745 & 0.1434 & 0.0000 & 0.3506 & 0.0000 & 0.0000 \\
INSTI & 7745 & 0.0499 & 0.0000 & 0.1404 & 0.0000 & 0.0000 \\
STD_NET_HIRE & 7745 & 0.1922 & 0.1000 & 0.3346 & 0.0527 & 0.1925 \\
\hline
\end{tabular}

Notes: Table 2 presents the descriptive statistics of the variables in our main regression model (Model (2)). See Appendix A for variable definitions.

Table 3 illustrates the Pearson correlations among variables, including an absolute value of abnormal net hiring and four variables of analysts' group affiliation. The table shows that there is a negative correlation between the total number of analysts and inefficacy in labor investment, indicating that there exists an analyst governance role. However, the correlations between the absolute value of abnormal net hiring and analysts' group affiliation are negative and significant (except for NGAGF), suggesting that a negative relation applies to almost all types of analysts. We, therefore, conclude that the effect of different group affiliation of analysts on firms' labor efficiency is not clear through correlations. A firm's labor investment efficiency has a positive correlation with firm size (SIZE), dividend payout ratio (DIVDUM), tangible asset ratio (TANGIBLE), and volatility of cash flow from operations and sales revenues (STD_CFO, STD_SALE). On the other hand, it has a negative correlation with market-to-book ratio (MTB), quick ratio (Quick), being a loss firm (LOSS), and the standard deviation of net hiring (STD_NET_HIRE). 
Table 3. Correlations.

\begin{tabular}{|c|c|c|c|c|c|c|c|c|c|c|c|c|c|c|c|c|c|c|}
\hline$\#$ & Variables & 1 & 2 & 3 & 4 & 5 & 6 & 7 & 8 & 9 & 10 & 11 & 12 & 13 & 14 & 15 & 16 & 17 \\
\hline 1 & $\left|A B \_N E T \_H I R E\right|$ & 1.00 & $\begin{array}{l}-0.05 \\
(0.00)\end{array}$ & $\begin{array}{l}-0.03 \\
(0.00)\end{array}$ & $\begin{array}{l}-0.04 \\
(0.00)\end{array}$ & $\begin{array}{l}-0.08 \\
(0.00)\end{array}$ & $\begin{array}{c}0.01 \\
(0.32)\end{array}$ & $\begin{array}{c}0.03 \\
(0.02)\end{array}$ & $\begin{array}{l}-0.07 \\
(0.00)\end{array}$ & $\begin{array}{c}0.05 \\
(0.00)\end{array}$ & $\begin{array}{c}0.02 \\
(0.11)\end{array}$ & $\begin{array}{l}-0.16 \\
(0.00)\end{array}$ & $\begin{array}{l}-0.05 \\
(0.00)\end{array}$ & $\begin{array}{l}-0.03 \\
(0.00)\end{array}$ & $\begin{array}{l}-0.09 \\
(0.00)\end{array}$ & $\begin{array}{c}0.10 \\
(0.00)\end{array}$ & $\begin{array}{l}-0.02 \\
(0.12)\end{array}$ & $\begin{array}{c}0.17 \\
(0.00)\end{array}$ \\
\hline 2 & Analyst following & $\begin{array}{l}-0.05 \\
(0.00) \\
\end{array}$ & 1.00 & $\begin{array}{c}0.32 \\
(0.00) \\
\end{array}$ & $\begin{array}{c}0.94 \\
(0.00) \\
\end{array}$ & $\begin{array}{c}0.70 \\
(0.00) \\
\end{array}$ & $\begin{array}{c}0.43 \\
(0.00) \\
\end{array}$ & $\begin{array}{c}0.03 \\
(0.01) \\
\end{array}$ & $\begin{array}{c}0.68 \\
(0.00) \\
\end{array}$ & $\begin{array}{l}-0.12 \\
(0.00) \\
\end{array}$ & $\begin{array}{c}0.25 \\
(0.00) \\
\end{array}$ & $\begin{array}{c}0.13 \\
(0.00) \\
\end{array}$ & $\begin{array}{c}0.41 \\
(0.00) \\
\end{array}$ & $\begin{array}{c}0.33 \\
(0.00) \\
\end{array}$ & $\begin{array}{c}0.13 \\
(0.00) \\
\end{array}$ & $\begin{array}{l}-0.08 \\
(0.00) \\
\end{array}$ & $\begin{array}{c}0.18 \\
(0.00) \\
\end{array}$ & $\begin{array}{l}-0.06 \\
(0.00) \\
\end{array}$ \\
\hline 3 & GAGF & $\begin{array}{l}-0.03 \\
(0.00)\end{array}$ & $\begin{array}{c}0.32 \\
(0.00)\end{array}$ & 1.00 & $\begin{array}{c}0.28 \\
(0.00)\end{array}$ & $\begin{array}{c}0.42 \\
(0.00) \\
\end{array}$ & $\begin{array}{l}-0.12 \\
(0.00)\end{array}$ & $\begin{array}{c}0.00 \\
(0.84)\end{array}$ & $\begin{array}{c}0.26 \\
(0.00)\end{array}$ & $\begin{array}{l}-0.06 \\
(0.00)\end{array}$ & $\begin{array}{c}0.10 \\
(0.00)\end{array}$ & $\begin{array}{c}0.04 \\
(0.00)\end{array}$ & $\begin{array}{c}0.30 \\
(0.00)\end{array}$ & $\begin{array}{c}0.24 \\
(0.00)\end{array}$ & $\begin{array}{c}0.02 \\
(0.08)\end{array}$ & $\begin{array}{l}-0.02 \\
(0.13)\end{array}$ & $\begin{array}{c}0.11 \\
(0.00)\end{array}$ & $\begin{array}{l}-0.04 \\
(0.00) \\
\end{array}$ \\
\hline 4 & GANGF & $\begin{array}{l}-0.04 \\
(0.00)\end{array}$ & $\begin{array}{c}0.94 \\
(0.00)\end{array}$ & $\begin{array}{c}0.28 \\
(0.00)\end{array}$ & 1.00 & $\begin{array}{c}0.63 \\
(0.00)\end{array}$ & $\begin{array}{c}0.29 \\
(0.00)\end{array}$ & $\begin{array}{c}0.02 \\
(0.08)\end{array}$ & $\begin{array}{c}0.59 \\
(0.00)\end{array}$ & $\begin{array}{l}-0.11 \\
(0.00)\end{array}$ & $\begin{array}{c}0.23 \\
(0.00)\end{array}$ & $\begin{array}{c}0.12 \\
(0.00)\end{array}$ & $\begin{array}{c}0.35 \\
(0.00)\end{array}$ & $\begin{array}{c}0.28 \\
(0.00)\end{array}$ & $\begin{array}{c}0.13 \\
(0.00)\end{array}$ & $\begin{array}{l}-0.07 \\
(0.00)\end{array}$ & $\begin{array}{c}0.22 \\
(0.00)\end{array}$ & $\begin{array}{l}-0.05 \\
(0.00) \\
\end{array}$ \\
\hline 5 & NGAGF & $\begin{array}{l}-0.08 \\
(0.00)\end{array}$ & $\begin{array}{c}0.70 \\
(0.00)\end{array}$ & $\begin{array}{c}0.42 \\
(0.00)\end{array}$ & $\begin{array}{c}0.63 \\
(0.00)\end{array}$ & 1.00 & $\begin{array}{l}-0.26 \\
(0.00)\end{array}$ & $\begin{array}{l}-0.01 \\
(0.54)\end{array}$ & $\begin{array}{c}0.61 \\
(0.00)\end{array}$ & $\begin{array}{l}-0.13 \\
(0.00)\end{array}$ & $\begin{array}{c}0.28 \\
(0.00)\end{array}$ & $\begin{array}{c}0.08 \\
(0.00)\end{array}$ & $\begin{array}{c}0.49 \\
(0.00)\end{array}$ & $\begin{array}{c}0.40 \\
(0.00)\end{array}$ & $\begin{array}{c}0.12 \\
(0.00)\end{array}$ & $\begin{array}{l}-0.02 \\
(0.18)\end{array}$ & $\begin{array}{c}0.07 \\
(0.00)\end{array}$ & $\begin{array}{l}-0.07 \\
(0.00)\end{array}$ \\
\hline 6 & NGANGF & $\begin{array}{c}0.01 \\
(0.32)\end{array}$ & $\begin{array}{c}0.43 \\
(0.00)\end{array}$ & $\begin{array}{l}-0.12 \\
(0.00)\end{array}$ & $\begin{array}{c}0.29 \\
(0.00)\end{array}$ & $\begin{array}{l}-0.26 \\
(0.00)\end{array}$ & 1.00 & $\begin{array}{c}0.05 \\
(0.00)\end{array}$ & $\begin{array}{c}0.20 \\
(0.00)\end{array}$ & $\begin{array}{c}0.00 \\
(0.67)\end{array}$ & $\begin{array}{l}-0.01 \\
(0.29) \\
\end{array}$ & $\begin{array}{c}0.06 \\
(0.00)\end{array}$ & $\begin{array}{l}-0.01 \\
(0.64)\end{array}$ & $\begin{array}{l}-0.03 \\
(0.01)\end{array}$ & $\begin{array}{c}0.00 \\
(0.81)\end{array}$ & $\begin{array}{l}-0.10 \\
(0.00)\end{array}$ & $\begin{array}{c}0.06 \\
(0.00)\end{array}$ & $\begin{array}{c}0.00 \\
(0.79) \\
\end{array}$ \\
\hline 7 & MTB & $\begin{array}{c}0.03 \\
(0.02) \\
\end{array}$ & $\begin{array}{c}0.03 \\
(0.01) \\
\end{array}$ & $\begin{array}{c}0.00 \\
(0.84) \\
\end{array}$ & $\begin{array}{c}0.02 \\
(0.08) \\
\end{array}$ & $\begin{array}{l}-0.01 \\
(0.54) \\
\end{array}$ & $\begin{array}{c}0.05 \\
(0.00) \\
\end{array}$ & 1.00 & $\begin{array}{c}0.07 \\
(0.00) \\
\end{array}$ & $\begin{array}{c}0.01 \\
(0.31) \\
\end{array}$ & $\begin{array}{c}0.00 \\
(0.77) \\
\end{array}$ & $\begin{array}{l}-0.06 \\
(0.00) \\
\end{array}$ & $\begin{array}{l}-0.01 \\
(0.22) \\
\end{array}$ & $\begin{array}{l}-0.01 \\
(0.26) \\
\end{array}$ & $\begin{array}{l}-0.06 \\
(0.00) \\
\end{array}$ & $\begin{array}{c}0.02 \\
(0.04) \\
\end{array}$ & $\begin{array}{l}-0.05 \\
(0.00) \\
\end{array}$ & $\begin{array}{c}0.03 \\
(0.01) \\
\end{array}$ \\
\hline 8 & SIZE & $\begin{array}{l}-0.07 \\
(0.00) \\
\end{array}$ & $\begin{array}{c}0.68 \\
(0.00) \\
\end{array}$ & $\begin{array}{c}0.26 \\
(0.00) \\
\end{array}$ & $\begin{array}{c}0.59 \\
(0.00) \\
\end{array}$ & $\begin{array}{c}0.61 \\
(0.00) \\
\end{array}$ & $\begin{array}{c}0.20 \\
(0.00) \\
\end{array}$ & $\begin{array}{c}0.07 \\
(0.00) \\
\end{array}$ & 1.00 & $\begin{array}{l}-0.13 \\
(0.00) \\
\end{array}$ & $\begin{array}{c}0.26 \\
(0.00) \\
\end{array}$ & $\begin{array}{c}0.15 \\
(0.00)\end{array}$ & $\begin{array}{c}0.48 \\
(0.00) \\
\end{array}$ & $\begin{array}{c}0.41 \\
(0.00) \\
\end{array}$ & $\begin{array}{c}0.10 \\
(0.00) \\
\end{array}$ & $\begin{array}{l}-0.11 \\
(0.00) \\
\end{array}$ & $\begin{array}{l}-0.10 \\
(0.00) \\
\end{array}$ & $\begin{array}{l}-0.07 \\
(0.00) \\
\end{array}$ \\
\hline 9 & Quick & $\begin{array}{c}0.05 \\
(0.00)\end{array}$ & $\begin{array}{l}-0.12 \\
(0.00)\end{array}$ & $\begin{array}{l}-0.06 \\
(0.00)\end{array}$ & $\begin{array}{l}-0.11 \\
(0.00)\end{array}$ & $\begin{array}{l}-0.13 \\
(0.00)\end{array}$ & $\begin{array}{c}0.00 \\
(0.67)\end{array}$ & $\begin{array}{c}0.01 \\
(0.31)\end{array}$ & $\begin{array}{l}-0.13 \\
(0.00)\end{array}$ & 1.00 & $\begin{array}{l}-0.12 \\
(0.00)\end{array}$ & $\begin{array}{c}0.01 \\
(0.60)\end{array}$ & $\begin{array}{l}-0.09 \\
(0.00)\end{array}$ & $\begin{array}{l}-0.07 \\
(0.00)\end{array}$ & $\begin{array}{l}-0.27 \\
(0.00)\end{array}$ & $\begin{array}{l}-0.05 \\
(0.00)\end{array}$ & $\begin{array}{l}-0.07 \\
(0.00)\end{array}$ & $\begin{array}{c}0.02 \\
(0.13)\end{array}$ \\
\hline 10 & LEV & $\begin{array}{c}0.02 \\
(0.11) \\
\end{array}$ & $\begin{array}{c}0.25 \\
(0.00) \\
\end{array}$ & $\begin{array}{c}0.10 \\
(0.00) \\
\end{array}$ & $\begin{array}{c}0.23 \\
(0.00) \\
\end{array}$ & $\begin{array}{c}0.28 \\
(0.00) \\
\end{array}$ & $\begin{array}{l}-0.01 \\
(0.29) \\
\end{array}$ & $\begin{array}{c}0.00 \\
(0.77) \\
\end{array}$ & $\begin{array}{c}0.26 \\
(0.00) \\
\end{array}$ & $\begin{array}{c}-0.12 \\
(0.00) \\
\end{array}$ & 1.00 & $\begin{array}{r}-0.09 \\
(0.00) \\
\end{array}$ & $\begin{array}{c}0.21 \\
(0.00) \\
\end{array}$ & $\begin{array}{c}0.18 \\
(0.00) \\
\end{array}$ & $\begin{array}{c}0.18 \\
(0.00) \\
\end{array}$ & $\begin{array}{c}0.14 \\
(0.00) \\
\end{array}$ & $\begin{array}{c}0.11 \\
(0.00) \\
\end{array}$ & $\begin{array}{c}0.01 \\
(0.44) \\
\end{array}$ \\
\hline 11 & DIVDUM & $\begin{array}{l}-0.16 \\
(0.00) \\
\end{array}$ & $\begin{array}{c}0.13 \\
(0.00) \\
\end{array}$ & $\begin{array}{c}0.04 \\
(0.00) \\
\end{array}$ & $\begin{array}{c}0.12 \\
(0.00) \\
\end{array}$ & $\begin{array}{c}0.08 \\
(0.00) \\
\end{array}$ & $\begin{array}{c}0.06 \\
(0.00) \\
\end{array}$ & $\begin{array}{l}-0.06 \\
(0.00) \\
\end{array}$ & $\begin{array}{c}0.15 \\
(0.00) \\
\end{array}$ & $\begin{array}{c}0.01 \\
(0.60) \\
\end{array}$ & $\begin{array}{l}-0.09 \\
(0.00) \\
\end{array}$ & 1.00 & $\begin{array}{c}0.04 \\
(0.00) \\
\end{array}$ & $\begin{array}{c}0.06 \\
(0.00) \\
\end{array}$ & $\begin{array}{c}0.06 \\
(0.00) \\
\end{array}$ & $\begin{array}{l}-0.48 \\
(0.00) \\
\end{array}$ & $\begin{array}{c}0.02 \\
(0.14) \\
\end{array}$ & $\begin{array}{l}-0.18 \\
(0.00) \\
\end{array}$ \\
\hline 12 & STD_CFO & $\begin{array}{l}-0.05 \\
(0.00) \\
\end{array}$ & $\begin{array}{c}0.41 \\
(0.00) \\
\end{array}$ & $\begin{array}{c}0.30 \\
(0.00) \\
\end{array}$ & $\begin{array}{c}0.35 \\
(0.00) \\
\end{array}$ & $\begin{array}{c}0.49 \\
(0.00) \\
\end{array}$ & $\begin{array}{l}-0.01 \\
(0.64) \\
\end{array}$ & $\begin{array}{l}-0.01 \\
(0.22) \\
\end{array}$ & $\begin{array}{c}0.48 \\
(0.00) \\
\end{array}$ & $\begin{array}{r}-0.09 \\
(0.00) \\
\end{array}$ & $\begin{array}{c}0.21 \\
(0.00) \\
\end{array}$ & $\begin{array}{c}0.04 \\
(0.00) \\
\end{array}$ & 1.00 & $\begin{array}{c}0.75 \\
(0.00) \\
\end{array}$ & $\begin{array}{c}0.06 \\
(0.00) \\
\end{array}$ & $\begin{array}{c}0.00 \\
(0.78) \\
\end{array}$ & $\begin{array}{c}0.00 \\
(0.77) \\
\end{array}$ & $\begin{array}{l}-0.05 \\
(0.00) \\
\end{array}$ \\
\hline 13 & STD_SALE & $\begin{array}{l}-0.03 \\
(0.00)\end{array}$ & $\begin{array}{c}0.33 \\
(0.00)\end{array}$ & $\begin{array}{c}0.24 \\
(0.00)\end{array}$ & $\begin{array}{c}0.28 \\
(0.00)\end{array}$ & $\begin{array}{c}0.40 \\
(0.00)\end{array}$ & $\begin{array}{c}-0.03 \\
(0.01)\end{array}$ & $\begin{array}{l}-0.01 \\
(0.26)\end{array}$ & $\begin{array}{c}0.41 \\
(0.00)\end{array}$ & $\begin{array}{l}-0.07 \\
(0.00)\end{array}$ & $\begin{array}{c}0.18 \\
(0.00)\end{array}$ & $\begin{array}{c}0.06 \\
(0.00)\end{array}$ & $\begin{array}{c}0.75 \\
(0.00)\end{array}$ & 1.00 & $\begin{array}{c}0.01 \\
(0.32)\end{array}$ & $\begin{array}{l}-0.02 \\
(0.14)\end{array}$ & $\begin{array}{l}-0.01 \\
(0.61)\end{array}$ & $\begin{array}{l}-0.03 \\
(0.03) \\
\end{array}$ \\
\hline 14 & TANGIBLE & $\begin{array}{l}-0.09 \\
(0.00)\end{array}$ & $\begin{array}{c}0.13 \\
(0.00)\end{array}$ & $\begin{array}{c}0.02 \\
(0.08)\end{array}$ & $\begin{array}{c}0.13 \\
(0.00)\end{array}$ & $\begin{array}{c}0.12 \\
(0.00)\end{array}$ & $\begin{array}{c}0.00 \\
(0.81)\end{array}$ & $\begin{array}{l}-0.06 \\
(0.00)\end{array}$ & $\begin{array}{c}0.10 \\
(0.00)\end{array}$ & $\begin{array}{l}-0.27 \\
(0.00)\end{array}$ & $\begin{array}{c}0.18 \\
(0.00)\end{array}$ & $\begin{array}{c}0.06 \\
(0.00)\end{array}$ & $\begin{array}{c}0.06 \\
(0.00)\end{array}$ & $\begin{array}{c}0.01 \\
(0.32)\end{array}$ & 1.00 & $\begin{array}{c}0.02 \\
(0.07)\end{array}$ & $\begin{array}{c}0.15 \\
(0.00)\end{array}$ & $\begin{array}{l}-0.09 \\
(0.00) \\
\end{array}$ \\
\hline 15 & LOSS & $\begin{array}{c}0.10 \\
(0.00)\end{array}$ & $\begin{array}{l}-0.08 \\
(0.00)\end{array}$ & $\begin{array}{l}-0.02 \\
(0.13)\end{array}$ & $\begin{array}{l}-0.07 \\
(0.00)\end{array}$ & $\begin{array}{l}-0.02 \\
(0.18)\end{array}$ & $\begin{array}{l}-0.10 \\
(0.00)\end{array}$ & $\begin{array}{c}0.02 \\
(0.04)\end{array}$ & $\begin{array}{l}-0.11 \\
(0.00)\end{array}$ & $\begin{array}{l}-0.05 \\
(0.00)\end{array}$ & $\begin{array}{c}0.14 \\
(0.00)\end{array}$ & $\begin{array}{l}-0.48 \\
(0.00)\end{array}$ & $\begin{array}{c}0.00 \\
(0.78)\end{array}$ & $\begin{array}{l}-0.02 \\
(0.14)\end{array}$ & $\begin{array}{c}0.02 \\
(0.07)\end{array}$ & 1.00 & $\begin{array}{c}0.03 \\
(0.02)\end{array}$ & $\begin{array}{c}0.08 \\
(0.00) \\
\end{array}$ \\
\hline 16 & INSTI & $\begin{array}{l}-0.02 \\
(0.12) \\
\end{array}$ & $\begin{array}{c}0.18 \\
(0.00)\end{array}$ & $\begin{array}{c}0.11 \\
(0.00)\end{array}$ & $\begin{array}{c}0.22 \\
(0.00) \\
\end{array}$ & $\begin{array}{c}0.07 \\
(0.00) \\
\end{array}$ & $\begin{array}{c}0.06 \\
(0.00)\end{array}$ & $\begin{array}{l}-0.05 \\
(0.00) \\
\end{array}$ & $\begin{array}{l}-0.10 \\
(0.00)\end{array}$ & $\begin{array}{l}-0.07 \\
(0.00) \\
\end{array}$ & $\begin{array}{c}0.11 \\
(0.00)\end{array}$ & $\begin{array}{c}0.02 \\
(0.14) \\
\end{array}$ & $\begin{array}{c}0.00 \\
(0.77)\end{array}$ & $\begin{array}{l}-0.01 \\
(0.61) \\
\end{array}$ & $\begin{array}{c}0.15 \\
(0.00) \\
\end{array}$ & $\begin{array}{c}0.03 \\
(0.02)\end{array}$ & 1.00 & $\begin{array}{l}-0.02 \\
(0.12)\end{array}$ \\
\hline 17 & STD_NET_HIRE & $\begin{array}{c}0.17 \\
(0.00)\end{array}$ & $\begin{array}{l}-0.06 \\
(0.00)\end{array}$ & $\begin{array}{l}-0.04 \\
(0.00)\end{array}$ & $\begin{array}{l}-0.05 \\
(0.00)\end{array}$ & $\begin{array}{l}-0.07 \\
(0.00)\end{array}$ & $\begin{array}{c}0.00 \\
(0.79)\end{array}$ & $\begin{array}{c}0.03 \\
(0.01)\end{array}$ & $\begin{array}{l}-0.07 \\
(0.00)\end{array}$ & $\begin{array}{c}0.02 \\
(0.13)\end{array}$ & $\begin{array}{c}0.01 \\
(0.44)\end{array}$ & $\begin{array}{l}-0.18 \\
(0.00)\end{array}$ & $\begin{array}{l}-0.05 \\
(0.00)\end{array}$ & $\begin{array}{l}-0.03 \\
(0.03)\end{array}$ & $\begin{array}{l}-0.09 \\
(0.00)\end{array}$ & $\begin{array}{c}0.08 \\
(0.00)\end{array}$ & $\begin{array}{l}-0.02 \\
(0.12)\end{array}$ & 1.00 \\
\hline
\end{tabular}

Notes: Table 3 presents the Pearson correlation matrix of the variables in our main regression model (Model (2)). $p$-values are presented in parentheses. See Appendix A for variable definitions. 


\subsection{Regression Results}

Using Model (2), we examined the association between analysts' group affiliation and a firm's abnormal net hiring. The results are reported in Table 4 . The regression result using a full sample is shown in column (1). The coefficient of NGAGF is negative and significant $(-0.0019 ; p$-value $<0.05)$, while the coefficients of GAGF, GANGF, and NGANGF are insignificant. This result indicates that labor investment efficiency increases in group firms (chaebols) as there are more nongroup analysts following, but it is not affected by group-affiliated analysts. Analysts' independency, therefore, positively affects the efficiency of firms' labor investment decisions, in addition to its impact on the quality of earnings forecasts found in prior studies [11,13]. Coefficients on control variables suggest that abnormal net hiring decreases when a firm paid dividends (DIVDUM) or had fewer tangible assets (TANGIBLE) in the prior year. On the other hand, abnormal net hiring increases when a firm had a loss (LOSS), more quick assets (Quick), or higher volatility in net hiring (STD_NET_HIRE) in a prior year.

Table 4. The effect of analyst following on abnormal net hiring.

\begin{tabular}{|c|c|c|c|c|c|c|}
\hline \multirow{4}{*}{$\begin{array}{c}\text { Independent Variables } \\
\text { Intercept }\end{array}$} & \multicolumn{6}{|c|}{ Dependent Variable: $\left|A B \_N E T \_H I R E\right|$} \\
\hline & \multicolumn{2}{|c|}{ Full Sample } & \multicolumn{2}{|c|}{ AB_NET_HIRE $>0$} & \multicolumn{2}{|c|}{$A B \_N E T \_H I R E<0$} \\
\hline & \multicolumn{2}{|c|}{ (1) } & \multicolumn{2}{|c|}{ (2) } & \multicolumn{2}{|c|}{ (3) } \\
\hline & $\begin{array}{c}0.3483 \\
(8.76)\end{array}$ & $* * *$ & $\begin{array}{l}0.9784 \\
(13.98)\end{array}$ & $* * *$ & $\begin{array}{c}0.1393 \\
(3.26)\end{array}$ & $* * *$ \\
\hline GAGF & $\begin{array}{c}-0.0033 \\
(-0.61)\end{array}$ & & $\begin{array}{c}-0.0013 \\
(-0.17)\end{array}$ & & $\begin{array}{c}-0.0086 \\
(-1.35)\end{array}$ & \\
\hline GANGF & $\begin{array}{c}0.0006 \\
(0.67)\end{array}$ & & $\begin{array}{c}-0.0001 \\
(-0.06)\end{array}$ & & $\begin{array}{c}0.0003 \\
(0.27)\end{array}$ & \\
\hline NGAGF & $\begin{array}{l}-0.0019 \\
(-1.98)\end{array}$ & $* *$ & $\begin{array}{l}0.0002 \\
(0.12)\end{array}$ & & $\begin{array}{l}-0.0029 \\
(-2.94)\end{array}$ & $* * *$ \\
\hline NGANGF & $\begin{array}{l}-0.0008 \\
(-0.98)\end{array}$ & & $\begin{array}{c}-0.0008 \\
(-0.59)\end{array}$ & & $\begin{array}{c}-0.0011 \\
(-1.27)\end{array}$ & \\
\hline MTB & $\begin{array}{l}0.3882 \\
(0.98)\end{array}$ & & $\begin{array}{c}9.7148 \\
(3.33)\end{array}$ & $* * *$ & $\begin{array}{c}0.1766 \\
(1.75)\end{array}$ & * \\
\hline SIZE & $\begin{array}{l}-0.0011 \\
(-0.44)\end{array}$ & & $\begin{array}{c}-0.0113 \\
(-2.30)\end{array}$ & ** & $\begin{array}{l}0.0056 \\
(2.05)\end{array}$ & ** \\
\hline Quick & $\begin{array}{l}0.0041 \\
(1.87)\end{array}$ & * & $\begin{array}{c}0.0019 \\
(0.44)\end{array}$ & & $\begin{array}{l}0.0064 \\
(3.10)\end{array}$ & $* * *$ \\
\hline$L E V$ & $\begin{array}{c}0.0895 \\
(1.87)\end{array}$ & * & $\begin{array}{l}0.1259 \\
(1.56)\end{array}$ & & $\begin{array}{l}0.0562 \\
(1.12)\end{array}$ & \\
\hline DIVDUM & $\begin{array}{l}-0.0421 \\
(-7.12)\end{array}$ & $* * *$ & $\begin{array}{c}-0.0375 \\
(-3.79)\end{array}$ & $* * *$ & $\begin{array}{l}-0.0393 \\
(-6.29)\end{array}$ & $* * *$ \\
\hline STD_CFO & $\begin{array}{l}0.0000 \\
(-0.98)\end{array}$ & & $\begin{array}{c}0.0000 \\
(1.17)\end{array}$ & & $\begin{array}{l}0.0000 \\
(-1.49)\end{array}$ & \\
\hline STD_SALE & $\begin{array}{l}0.0000 \\
(-0.76)\end{array}$ & & $\begin{array}{l}0.0000 \\
(-2.14)\end{array}$ & ** & $\begin{array}{l}0.0000 \\
(0.87)\end{array}$ & \\
\hline TANGIBLE & $\begin{array}{l}-0.0346 \\
(-2.10)\end{array}$ & ** & $\begin{array}{c}-0.0453 \\
(-1.73)\end{array}$ & * & $\begin{array}{l}-0.0169 \\
(-1.05)\end{array}$ & \\
\hline LOSS & $\begin{array}{l}0.0162 \\
(2.29)\end{array}$ & ** & $\begin{array}{c}0.0204 \\
(1.57)\end{array}$ & & $\begin{array}{l}0.0217 \\
(2.91)\end{array}$ & $* * *$ \\
\hline INSTI & $\begin{array}{c}-0.0341 \\
(-1.45)\end{array}$ & & $\begin{array}{c}-0.0205 \\
(-0.47)\end{array}$ & & $\begin{array}{c}-0.0298 \\
(-1.27)\end{array}$ & \\
\hline
\end{tabular}


Table 4. Cont.

\begin{tabular}{|c|c|c|c|c|c|c|}
\hline & \multicolumn{6}{|c|}{ Dependent Variable: $\left|A B \_N E T \_H I R E\right|$} \\
\hline & \multicolumn{2}{|c|}{ Full Sample } & \multicolumn{2}{|c|}{$A B \_N E T \_H I R E>0$} & \multicolumn{2}{|c|}{$A B \_N E T \_H I R E<0$} \\
\hline Independent Variables & \multicolumn{2}{|c|}{ (1) } & \multicolumn{2}{|c|}{ (2) } & \multicolumn{2}{|c|}{ (3) } \\
\hline STD_NET_HIRE & $\begin{array}{c}0.0582 \\
(6.25)\end{array}$ & $* * *$ & $\begin{array}{c}0.0742 \\
(4.68)\end{array}$ & $* * *$ & $\begin{array}{c}0.0390 \\
(3.88)\end{array}$ & $* * *$ \\
\hline Year-fixed effect & Yes & & Yes & & Yes & \\
\hline Industry-fixed effect & Yes & & Yes & & Yes & \\
\hline [F-value] & [21.47] & $* * *$ & [10.09] & $* * *$ & [16.63] & $* * *$ \\
\hline $\mathrm{R}^{2}$ & 0.092 & & 0.113 & & 0.134 & \\
\hline $\mathrm{N}$ & 7745 & & 3447 & & 4298 & \\
\hline
\end{tabular}

Notes: Table 4 presents the regression results of abnormal net hiring ( $\left|A B_{-} N E T \_H I R E\right|$ or $\left.A B_{-} N E T_{-} H I R E\right)$ on analysts' group affiliation (GAGF, GANGF, NGAGF, NGANGF) and control variables. Column (1) reports regression results when an absolute value of abnormal net hiring ( $\left.\left|A B_{-} N E T_{-} H I R E\right|\right)$ is used as the dependent variable, and Columns (2) and (3) report results using a signed abnormal net hiring (AB_NET_HIRE) as the dependent variable. Model (1) tests the full sample and Models (2) and (3) test two different subsamples having positive and negative abnormal net hiring, respectively. T-statistics are calculated based on robust standard errors clustered at the firm level and are reported in parentheses below the coefficient estimates. The F-value is reported in square brackets. Statistical significance at the 1,5 , and $10 \%$ levels is denoted by $* * * * *$, and *, respectively. See Appendix A for variable definitions.

Columns (2) and (3) report regression results using two different subsamples. In the full sample of 7745 firm-year observations, 3447 observations have positive abnormal net hiring, while 4298 observations have negative abnormal net hiring. Depending on whether $A B_{-} N E T \_H I R E_{i, t}$ is positive or negative, we separated the observations into two subsamples and defined overinvestment (underinvestment) groups as firms whose abnormal net hiring is above (below) zero. After running Model (2) for each of two subgroups, we find that the coefficients of GAGF, GANGF, and NGANGF are insignificant for both subsamples, suggesting that group-affiliated analysts and nongroup analysts in nongroup firms do not affect abnormal net hiring. However, while the coefficient of NGAGF is insignificant using the overinvestment group, it is negative and significant for the underinvestment group. Thus, we argue that unaffiliated analysts are the ones who are enhancing the labor investment efficiency of group firms by resolving underinvestment problems. Overall, our results support the conflict-of-interest theory rather than the information sharing theory.

A firm's net hiring captures changes in employees, which is the number of hired employees minus the number of fired employees. Following Jung, Lee, and Weber [17], we formed four groups of firms based on the expected difference between the number of hired employees and the number of fired employees: (1) The over-hiring group consists of firms who are overinvesting while positive net hiring is expected (abnormal net hiring and expected net hiring are positive); (2) The under-firing group consists of firms who are overinvesting while negative net hiring is expected (abnormal net hiring is positive and expected net hiring is negative); (3) The under-hiring group consists of firms who are underinvesting while positive net hiring is expected (abnormal net hiring is negative and expected net hiring is positive); (4) The over-firing group consists of firms who are underinvesting while negative net hiring is expected (abnormal net hiring and expected net hiring is negative). Out of the total sample, the proportions of these four groups are $42,2,19$, and $37 \%$, respectively.

Using four subsamples of companies with different types of labor investment inefficiencies, we tested the effect of analysts' group affiliation. As presented in Table 5, the coefficient of NGAGF is negative and significant only in the over-firing sample. This indicates that our results regarding a positive effect of nongroup analysts on group firms' labor investment efficiency hold in the over-firing sample. Thus, we conclude that unaffiliated analysts improve nongroup firms' labor investment efficiency by decreasing the over-firing problem, while analysts who work for group-affiliated firms do not positively affect labor investment efficiency. 
Table 5. The effect of analyst following on over- and under-hiring (and firing).

\begin{tabular}{|c|c|c|c|c|c|c|c|c|}
\hline & \multicolumn{8}{|c|}{ Dependent Variable: $\left|A B \_N E T \_H I R E\right|$} \\
\hline & \multicolumn{2}{|c|}{ Over-Hiring } & \multicolumn{2}{|c|}{ Under-Firing } & \multicolumn{2}{|c|}{ Under-Hiring } & \multicolumn{2}{|c|}{ Over-Firing } \\
\hline Independent Variables & \multicolumn{2}{|c|}{ (1) } & \multicolumn{2}{|c|}{ (2) } & \multicolumn{2}{|c|}{ (3) } & \multicolumn{2}{|c|}{ (4) } \\
\hline Intercept & $\begin{array}{l}0.9689 \\
(13.47)\end{array}$ & $* * *$ & $\begin{array}{c}0.0422 \\
(1.70)\end{array}$ & $*$ & $\begin{array}{c}0.2125 \\
(8.87)\end{array}$ & & $\begin{array}{c}0.1232 \\
(1.93)\end{array}$ & $*$ \\
\hline GAGF & $\begin{array}{c}-0.0028 \\
(-0.34)\end{array}$ & & $\begin{array}{l}0.0055 \\
(0.49)\end{array}$ & & $\begin{array}{c}0.0036 \\
(0.79)\end{array}$ & & $\begin{array}{c}-0.0109 \\
(-1.16)\end{array}$ & \\
\hline GANGF & $\begin{array}{c}-0.0002 \\
(-0.16)\end{array}$ & & $\begin{array}{c}0.0003 \\
(0.54)\end{array}$ & & $\begin{array}{c}0.0007 \\
(1.20)\end{array}$ & & $\begin{array}{c}0.0003 \\
(0.21)\end{array}$ & \\
\hline NGAGF & $\begin{array}{c}-0.0002 \\
(-0.13)\end{array}$ & & $\begin{array}{c}0.0002 \\
(0.23)\end{array}$ & & $\begin{array}{c}-0.0005 \\
(-0.83)\end{array}$ & & $\begin{array}{c}-0.0034 \\
(-2.24)\end{array}$ & $* *$ \\
\hline NGANGF & $\begin{array}{c}-0.0010 \\
(-0.77)\end{array}$ & & $\begin{array}{c}0.0009 \\
(1.30)\end{array}$ & & $\begin{array}{c}0.0002 \\
(0.47)\end{array}$ & & $\begin{array}{c}-0.0008 \\
(-0.59)\end{array}$ & \\
\hline MTB & $\begin{array}{l}9.2717 \\
(3.15)\end{array}$ & $* * *$ & $\begin{array}{c}3.2007 \\
(1.80)\end{array}$ & * & $\begin{array}{c}2.6103 \\
(2.30)\end{array}$ & & $\begin{array}{l}0.1570 \\
(1.10)\end{array}$ & \\
\hline SIZE & $\begin{array}{c}-0.0104 \\
(-2.08)\end{array}$ & $* *$ & $\begin{array}{c}-0.0034 \\
(-1.96)\end{array}$ & * & $\begin{array}{c}-0.0017 \\
(-1.05)\end{array}$ & & $\begin{array}{c}0.0083 \\
(2.17)\end{array}$ & $* *$ \\
\hline Quick & $\begin{array}{c}0.0013 \\
(0.30)\end{array}$ & & $\begin{array}{c}-0.0045 \\
(-0.92)\end{array}$ & & $\begin{array}{c}0.0050 \\
(2.87)\end{array}$ & & $\begin{array}{l}0.0100 \\
(2.45)\end{array}$ & $* *$ \\
\hline$L E V$ & $\begin{array}{l}0.1494 \\
(1.75)\end{array}$ & * & $\begin{array}{l}0.0048 \\
(0.27)\end{array}$ & & $\begin{array}{l}0.0581 \\
(1.60)\end{array}$ & & $\begin{array}{c}0.0389 \\
(0.61)\end{array}$ & \\
\hline DIVDUM & $\begin{array}{c}-0.0386 \\
(-3.74)\end{array}$ & $* * *$ & $\begin{array}{c}0.0040 \\
(0.91)\end{array}$ & & $\begin{array}{c}-0.0188 \\
(-3.73)\end{array}$ & & $\begin{array}{l}-0.0387 \\
(-4.94)\end{array}$ & $* * *$ \\
\hline STD_CFO & $\begin{array}{c}0.0000 \\
(1.40)\end{array}$ & & $\begin{array}{c}0.0000 \\
(0.61)\end{array}$ & & $\begin{array}{l}0.0000 \\
(-0.63)\end{array}$ & & $\begin{array}{l}0.0000 \\
(-0.96)\end{array}$ & \\
\hline STD_SALE & $\begin{array}{l}0.0000 \\
(-2.31)\end{array}$ & $* *$ & $\begin{array}{l}0.0000 \\
(-0.06)\end{array}$ & & $\begin{array}{l}0.0000 \\
(0.13)\end{array}$ & & $\begin{array}{c}0.0000 \\
(0.29)\end{array}$ & \\
\hline TANGIBLE & $\begin{array}{c}-0.0441 \\
(-1.61)\end{array}$ & & $\begin{array}{c}0.0003 \\
(0.02)\end{array}$ & & $\begin{array}{c}-0.0211 \\
(-2.02)\end{array}$ & & $\begin{array}{c}-0.0229 \\
(-1.02)\end{array}$ & \\
\hline LOSS & $\begin{array}{c}0.0302 \\
(2.14)\end{array}$ & ** & $\begin{array}{c}0.0036 \\
(0.75)\end{array}$ & & $\begin{array}{c}-0.0056 \\
(-0.79)\end{array}$ & & $\begin{array}{c}0.0119 \\
(1.33)\end{array}$ & \\
\hline INSTI & $\begin{array}{c}-0.0052 \\
(-0.11)\end{array}$ & & $\begin{array}{c}-0.0052 \\
(-0.41)\end{array}$ & & $\begin{array}{c}0.0119 \\
(0.88)\end{array}$ & & $\begin{array}{c}-0.0313 \\
(-1.06)\end{array}$ & \\
\hline STD_NET_HIRE & $\begin{array}{l}0.0742 \\
(4.60)\end{array}$ & $* * *$ & $\begin{array}{c}-0.0002 \\
(-0.07)\end{array}$ & & $\begin{array}{c}0.0055 \\
(0.82)\end{array}$ & & $\begin{array}{c}0.0409 \\
(3.09)\end{array}$ & $* * *$ \\
\hline Year-fixed effect & Yes & & Yes & & Yes & & Yes & \\
\hline Industry-fixed effect & Yes & & Yes & & Yes & & Yes & \\
\hline [F-value] & [10.55] & $* * *$ & [27.18] & $* * *$ & [342.62] & $* * *$ & [33.35] & $* * *$ \\
\hline$R^{2}$ & 0.114 & & 0.575 & & 0.252 & & 0.146 & \\
\hline $\mathrm{N}$ & 3316 & & 151 & & 1527 & & 2891 & \\
\hline
\end{tabular}

Notes: Table 5 reports the results of estimating Model (2) on four different subsamples. Over-hiring is the sample where both abnormal net hiring and expected net hiring are positive. Under-firing is the sample where abnormal net hiring is positive and expected net hiring is negative. Under-hiring is the sample where abnormal net hiring is negative and expected net hiring is positive. Over-hiring is the sample where abnormal net hiring is negative and expected net hiring is negative. T-statistics are calculated based on robust standard errors clustered at the firm level and are reported in parentheses below the coefficient estimates. The F-value is reported in square brackets. Statistical significance at the 1,5 , and $10 \%$ levels is denoted by $* * * * *$, and *, respectively. See Appendix A for variable definitions.

As shown in column (4), other factors such as firm size (SIZE), quick asset ratio (Quick), and volatility in net hiring (STD_NET_HIRE) increase over-firing, but dividend payments (DIVDUM) decreases over-firing. On the other hand, regarding the overinvestment problem, larger firms (SIZE), and firms with fewer growth opportunities $(M T B)$, leverage $(L E V)$, volatility in sales revenue (STD_SALE), and net 
hire (STD_NET_HIRE) decrease over-hiring. Also, loss firms (LOSS) or firms who paid dividends (DIVDUM) have less of an over-hiring problem. Lastly, firms' under-firing decreases for larger firms (SIZE) with smaller market-to-book ratio (MTB).

\section{Additional Tests}

\subsection{The Impact of Inside Fund on Labor Investment Efficiency}

We document a positive association between nongroup, unaffiliated analysts and labor investment efficiency in group firms. Although analysts can influence firms' employment decisions, it would not be possible for firms to change their employment policy without sufficient funds. Given that the cost of financing increases in the order of internal funds, debt, and equity (pecking order theory), financing through internal funds can induce more efficient investment. Therefore, we expect to see our main result hold when a firm has sufficient internal funds, which is measured by the level of cash and cash equivalents. A firm is considered to have a high (low) level of cash when its cash and cash equivalents scaled by total assets is above (below) the yearly median. We then re-estimated regression Model (2) by using these two subsamples.

Table 6 presents the regression results from this test of the effect of funds within the company on the relationship between nongroup analysts following and labor investment efficiency. As we predicted, the positive effect of nongroup analysts on group firms' labor investment efficiency holds only when firms have sufficient funds: the coefficient of NGAGF is negative and significant at the $10 \%$ level. The coefficient of NGAGF is negative but insignificant when using a subsample of firms with low cash levels. Thus, the result implies that the nongroup analysts' effect on the increase in labor investment efficiency becomes stronger when a firm has high cash holdings.

Table 6. The effect of inside funding on our hypothesized relationship.

\begin{tabular}{|c|c|c|c|c|}
\hline & \multicolumn{4}{|c|}{ Dependent Variable: $\left|A B \_N E T \_H I R E\right|$} \\
\hline & \multicolumn{2}{|c|}{ High Fund } & \multicolumn{2}{|c|}{ Low Fund } \\
\hline Independent Variables & \multicolumn{2}{|c|}{ (1) } & \multicolumn{2}{|c|}{ (2) } \\
\hline Intercept & $\begin{array}{c}0.3563 \\
(6.05)\end{array}$ & $* * *$ & $\begin{array}{c}0.3027 \\
(6.39)\end{array}$ & $* * *$ \\
\hline GAGF & $\begin{array}{c}-0.0069 \\
(-0.67)\end{array}$ & & $\begin{array}{c}0.0008 \\
(0.13)\end{array}$ & \\
\hline GANGF & $\begin{array}{c}0.0003 \\
(0.22)\end{array}$ & & $\begin{array}{c}0.0008 \\
(0.87)\end{array}$ & \\
\hline NGAGF & $\begin{array}{c}-0.0026 \\
(-1.76)\end{array}$ & * & $\begin{array}{c}-0.0013 \\
(-1.00)\end{array}$ & \\
\hline NGANGF & $\begin{array}{c}-0.0016 \\
(-1.38)\end{array}$ & & $\begin{array}{c}-0.0015 \\
(-1.36)\end{array}$ & \\
\hline MTB & $\begin{array}{c}6.8580 \\
(2.77)\end{array}$ & $* * *$ & $\begin{array}{c}0.1147 \\
(0.69)\end{array}$ & \\
\hline SIZE & $\begin{array}{c}-0.0013 \\
-0.31)\end{array}$ & & $\begin{array}{c}-0.0033 \\
(-1.10)\end{array}$ & \\
\hline Quick & $\begin{array}{c}0.0030 \\
(1.04)\end{array}$ & & $\begin{array}{c}0.0053 \\
(0.87)\end{array}$ & \\
\hline LEV & $\begin{array}{c}0.1744 \\
(2.14)\end{array}$ & ** & $\begin{array}{c}0.0522 \\
(0.96)\end{array}$ & \\
\hline DIVDUM & $\begin{array}{c}-0.0440 \\
(-4.72)\end{array}$ & $* * *$ & $\begin{array}{c}-0.0301 \\
(-3.75)\end{array}$ & $* * *$ \\
\hline
\end{tabular}


Table 6. Cont.

\begin{tabular}{|c|c|c|c|c|}
\hline & \multicolumn{4}{|c|}{ Dependent Variable: $\mid A B \_N E T \_H I R E$} \\
\hline & \multicolumn{2}{|c|}{ High Fund } & \multicolumn{2}{|c|}{ Low Fund } \\
\hline Independent Variables & \multicolumn{2}{|c|}{ (1) } & \multicolumn{2}{|c|}{ (2) } \\
\hline STD_CFO & $\begin{array}{c}0.0000 \\
(0.12)\end{array}$ & & $\begin{array}{l}0.0000 \\
(-1.07)\end{array}$ & \\
\hline STD_SALE & $\begin{array}{c}0.0000 \\
(-0.44)\end{array}$ & & $\begin{array}{c}0.0000 \\
(-0.13)\end{array}$ & \\
\hline TANGIBLE & $\begin{array}{c}-0.0450 \\
(-1.80)\end{array}$ & * & $\begin{array}{c}0.0154 \\
(0.72)\end{array}$ & \\
\hline LOSS & $\begin{array}{c}0.0469 \\
(3.73)\end{array}$ & $* * *$ & $\begin{array}{c}-0.0039 \\
(-0.57)\end{array}$ & \\
\hline INSTI & $\begin{array}{c}0.0044 \\
(0.09)\end{array}$ & & $\begin{array}{c}-0.0524 \\
(-2.41)\end{array}$ & $* *$ \\
\hline STD_NET_HIRE & $\begin{array}{c}0.0659 \\
(4.36)\end{array}$ & $* * *$ & $\begin{array}{c}0.0401 \\
(3.97)\end{array}$ & $* * *$ \\
\hline Year-fixed effect & Yes & & Yes & \\
\hline Industry-fixed effect & Yes & & Yes & \\
\hline [F-value] & [13.35] & $* * *$ & [153.16] & $* * *$ \\
\hline $\mathrm{R}^{2}$ & 0.122 & & 0.088 & \\
\hline $\mathrm{N}$ & 3873 & & 3872 & \\
\hline
\end{tabular}

Notes: Table 6 reports the results of estimating Model (2) on two different subsamples. We separated firms into two groups: one with greater inside funding and the other with lower inside funding. A firm is considered to have a greater (lower) cash holding if a firm's cash and cash equivalents are greater (less) than the sample yearly median. T-statistics are calculated based on robust standard errors clustered at the firm level and are reported in parentheses below the coefficient estimates. The F-value is reported in square brackets. Statistical significance at the 1,5 , and $10 \%$ levels is denoted by ${ }^{* * *},{ }^{* *}$, and ${ }^{*}$, respectively. See Appendix A for variable definitions.

\subsection{Sensitivity Test-Endogeneity}

We also conducted a test to ensure that our main finding is causal. Our main specification measures analysts following using five different factors (firm size, performance, growth rate, external financing, and volatility of cash flows), and then estimates the relationship between analysts following and labor investment efficiency using a 2SLS regression model. Following Yu (2008), five factors are selected to control for other factors that affect analyst coverage. In the first stage, we modeled the estimate for the number of analysts following as:

$$
\begin{aligned}
& G_{A G F_{i, t}} \text { or } G A N G F_{i, t} \text { or } N G A G F_{i, t} \text { or } N G A N G F_{i, t} \\
& =\alpha_{0}+\beta_{1} M K V_{i, t-1}+\beta_{2} R O A_{i, t-1}+\beta_{3} G R O W T H_{i, t-1} \\
& +\beta_{4} \text { EXFINACT }_{i, t-1}+\gamma_{1} \text { CFVolatility } y_{i, t-1}+\text { Year Fixed Effects }+\varepsilon_{i, t}
\end{aligned}
$$

where the dependent variable is one of the analysts' group affiliation variables; $M K V$ is the market value; $R O A$ is return on assets, which is measured by net income divided by total assets; GROWTH is the growth rate of total assets, calculated by dividing change in assets by last year's assets; EXFINACT is net cash proceeds from external financing scaled by total assets; and CFVolatility is a standard deviation of cash flow scaled by last year's total assets.

The residuals from regression Model (3) are used as independent variables of interest in the next step (regression Model (2)) to obtain the effect of analyst following that is uncorrelated with firm size, profitability, growth, external financing, and cash flow volatility. The results of the first and second step regressions are illustrated in Table 7 . The results of the first stage regression indicate that firm size $(M K V)$ is positively associated with analyst following. For group firms, cash flow volatility 
(CFVolatility) is negatively correlated with analyst following, and for nongroup firms, past profitability $(R O A)$ increases analyst following while external financing activities (EXFINACT) decreases analyst following. Our main interest is in the second-stage regression in which we estimate the effect of analyst following on abnormal net hiring. Consistent with the results in Table 4, none of the coefficients of group-affiliated analyst following (residual(GAGF), residual(GANGF)) are significant, indicating that affiliated analysts do not influence firms' labor investment efficiency. However, we find that nongroup unaffiliated analysts following decrease firms' abnormal net hiring regardless of whether following firms are group or nongroup. Specifically, both residual(NGAGF) and residual(NGANGF) have negative and significant coefficients. After addressing the potential endogeneity problem, we confirm that our main finding that nongroup analysts following increases firms' labor investment efficiency holds.

Table 7. Two-stage least squares regression (2SLS) regression.

\begin{tabular}{|c|c|c|c|c|c|c|c|c|}
\hline \multicolumn{9}{|c|}{ First Stage: Regression to Estimate Expected Level of Analyst Coverage } \\
\hline \multirow{3}{*}{$\begin{array}{c}\text { Independent Variables } \\
\text { Intercept }\end{array}$} & \multicolumn{8}{|c|}{ Dependent Variable: } \\
\hline & \multicolumn{2}{|c|}{ GAGF (1) } & \multicolumn{2}{|c|}{ GANGF (2) } & \multicolumn{2}{|c|}{ NGAGF (3) } & \multicolumn{2}{|c|}{ NGANGF (4) } \\
\hline & $\begin{array}{l}-0.027 \\
(-1.74)\end{array}$ & $*$ & $\begin{array}{l}-0.809 \\
(-3.57)\end{array}$ & $* * *$ & $\begin{array}{l}-0.455 \\
(-2.38)\end{array}$ & $* *$ & $\begin{array}{l}5.120 \\
(29.68)\end{array}$ & $* * *$ \\
\hline$M K V$ & $\begin{array}{l}0.000 \\
(24.15)\end{array}$ & $* * *$ & $\begin{array}{l}0.000 \\
(26.42)\end{array}$ & $* * *$ & $\begin{array}{c}0.000 \\
(33.97)\end{array}$ & $* * *$ & $\begin{array}{l}0.000 \\
(3.89)\end{array}$ & $* * *$ \\
\hline$R O A$ & $\begin{array}{l}-0.020 \\
(-0.75)\end{array}$ & & $\begin{array}{l}2.653 \\
(6.90)\end{array}$ & *** & $\begin{array}{l}-0.770 \\
(-2.37)\end{array}$ & ** & $\begin{array}{c}3.424 \\
(11.69)\end{array}$ & $* * *$ \\
\hline GROWTH & $\begin{array}{l}0.002 \\
(0.31)\end{array}$ & & $\begin{array}{l}0.018 \\
(0.16)\end{array}$ & & $\begin{array}{l}0.051 \\
(0.54)\end{array}$ & & $\begin{array}{l}0.002 \\
(0.02)\end{array}$ & \\
\hline EXFINACT & $\begin{array}{l}0.015 \\
(0.42)\end{array}$ & & $\begin{array}{l}-3.211 \\
(-6.04)\end{array}$ & $* * *$ & $\begin{array}{l}-0.485 \\
-1.08)\end{array}$ & & $\begin{array}{l}-2.877 \\
-7.11)\end{array}$ & $* * *$ \\
\hline CFVolatility & $\begin{array}{l}-0.058 \\
(-2.36)\end{array}$ & ** & $\begin{array}{l}-1.504 \\
(-4.14)\end{array}$ & $* * *$ & $\begin{array}{l}-2.051 \\
(-6.69)\end{array}$ & $* * *$ & $\begin{array}{l}0.581 \\
(2.10)\end{array}$ & ** \\
\hline Year fixed effects & Yes & & Yes & & Yes & & Yes & \\
\hline [F-value] & [31.85] & $* * *$ & [71.38] & $* * *$ & [64.75] & $* * *$ & [41.54] & $* * *$ \\
\hline$R^{2}$ & 0.0818 & & 0.1664 & & 0.1533 & & 0.104 & \\
\hline $\mathrm{N}$ & 7533 & & 7533 & & 7533 & & 7533 & \\
\hline \multicolumn{9}{|c|}{ Second Stage: Regression Using Residual Analyst Coverage } \\
\hline & & & \multicolumn{6}{|c|}{ Dependent Variable: $\left|A B \_N E T \_H I R E\right|$} \\
\hline & & & \multicolumn{2}{|c|}{ Full Sample } & \multicolumn{2}{|c|}{$A B \_N E T \_H I R E>0$} & \multicolumn{2}{|c|}{$A B \_N E T \_H I R E<0$} \\
\hline \multicolumn{3}{|c|}{ Independent Variables } & \multicolumn{2}{|c|}{ (1) } & \multicolumn{2}{|c|}{ (2) } & \multicolumn{2}{|c|}{ (3) } \\
\hline \multicolumn{3}{|c|}{ Intercept } & $\begin{array}{l}0.3500 \\
(9.07)\end{array}$ & $* * *$ & $\begin{array}{l}0.3500 \\
(9.07)\end{array}$ & $* * *$ & $\begin{array}{l}0.1518 \\
(3.59)\end{array}$ & $* * *$ \\
\hline \multicolumn{3}{|c|}{ residual(GAGF) } & $\begin{array}{l}-0.0037 \\
(-0.69)\end{array}$ & & $\begin{array}{l}-0.0037 \\
(-0.69)\end{array}$ & & $\begin{array}{c}-0.0081 \\
(-1.27)\end{array}$ & \\
\hline \multicolumn{3}{|c|}{ residual(GANGF) } & $\begin{array}{l}0.0007 \\
(0.85)\end{array}$ & & $\begin{array}{l}0.0007 \\
(0.85)\end{array}$ & & $\begin{array}{l}0.0007 \\
(0.76)\end{array}$ & \\
\hline \multicolumn{3}{|c|}{ residual(NGAGF) } & $\begin{array}{l}-0.0017 \\
(-1.75)\end{array}$ & $*$ & $\begin{array}{l}-0.0017 \\
(-1.75) \\
\end{array}$ & * & $\begin{array}{l}-0.0029 \\
(-3.12) \\
\end{array}$ & $* * *$ \\
\hline \multicolumn{3}{|c|}{ residual(NGANGF) } & $\begin{array}{l}-0.0015 \\
(-1.96)\end{array}$ & $*$ & $\begin{array}{l}-0.0015 \\
(-1.96) \\
\end{array}$ & * & $\begin{array}{l}-0.0015 \\
(-1.80) \\
\end{array}$ & * \\
\hline \multicolumn{3}{|l|}{ MTB } & $\begin{array}{l}0.3766 \\
(0.98)\end{array}$ & & $\begin{array}{l}0.3766 \\
(0.98)\end{array}$ & & $\begin{array}{l}0.1657 \\
(1.78)\end{array}$ & $*$ \\
\hline \multicolumn{3}{|l|}{ SIZE } & $\begin{array}{l}-0.0014 \\
(-0.60)\end{array}$ & & $\begin{array}{l}-0.0014 \\
(-0.60)\end{array}$ & & $\begin{array}{c}0.0044 \\
(1.70)\end{array}$ & $*$ \\
\hline \multicolumn{3}{|l|}{ Quick } & $\begin{array}{l}0.0041 \\
(1.68)\end{array}$ & $*$ & $\begin{array}{l}0.0041 \\
(1.68)\end{array}$ & * & $\begin{array}{l}0.0062 \\
(2.26)\end{array}$ & ** \\
\hline
\end{tabular}


Table 7. Cont.

\begin{tabular}{|c|c|c|c|c|c|c|}
\hline \multicolumn{7}{|c|}{ Second Stage: Regression Using Residual Analyst Coverage } \\
\hline & \multicolumn{6}{|c|}{ Dependent Variable: $\left|A B \_N E T \_H I R E\right|$} \\
\hline & \multicolumn{2}{|c|}{ Full Sample } & \multicolumn{2}{|c|}{$A B \_N E T \_H I R E>0$} & \multicolumn{2}{|c|}{$A B \_N E T \_H I R E<0$} \\
\hline Independent Variables & \multicolumn{2}{|c|}{ (1) } & \multicolumn{2}{|c|}{ (2) } & \multicolumn{2}{|c|}{ (3) } \\
\hline$L E V$ & $\begin{array}{l}0.0964 \\
(1.95)\end{array}$ & * & $\begin{array}{l}0.0964 \\
(1.95)\end{array}$ & * & $\begin{array}{l}0.0631 \\
(1.21)\end{array}$ & \\
\hline DIVDUM & $\begin{array}{l}-0.0434 \\
(-7.10)\end{array}$ & $* * *$ & $\begin{array}{l}-0.0434 \\
(-7.10)\end{array}$ & $* * *$ & $\begin{array}{l}-0.0395 \\
(-6.07)\end{array}$ & $* * *$ \\
\hline STD_CFO & $\begin{array}{l}0.0000 \\
(-1.78)\end{array}$ & * & $\begin{array}{l}0.0000 \\
-1.78)\end{array}$ & * & $\begin{array}{l}0.0000 \\
(-2.67)\end{array}$ & $* * *$ \\
\hline STD_SALE & $\begin{array}{l}0.0000 \\
(-0.95)\end{array}$ & & $\begin{array}{l}0.0000 \\
(-0.95)\end{array}$ & & $\begin{array}{l}0.0000 \\
(0.80)\end{array}$ & \\
\hline TANGIBLE & $\begin{array}{l}-0.0355 \\
(-2.11)\end{array}$ & ** & $\begin{array}{l}-0.0355 \\
(-2.11)\end{array}$ & $* *$ & $\begin{array}{c}-0.0206 \\
(-1.25)\end{array}$ & \\
\hline LOSS & $\begin{array}{l}0.0152 \\
(2.10)\end{array}$ & ** & $\begin{array}{l}0.0152 \\
(2.10)\end{array}$ & ** & $\begin{array}{l}0.0224 \\
(2.91)\end{array}$ & $* * *$ \\
\hline INSTI & $\begin{array}{l}-0.0358 \\
(-1.49)\end{array}$ & & $\begin{array}{l}-0.0358 \\
(-1.49)\end{array}$ & & $\begin{array}{l}-0.0309 \\
(-1.28)\end{array}$ & \\
\hline STD_NET_HIRE & $\begin{array}{l}0.0589 \\
(6.14)\end{array}$ & $* * *$ & $\begin{array}{l}0.0589 \\
(6.14)\end{array}$ & $* * *$ & $\begin{array}{l}0.0404 \\
(3.83)\end{array}$ & $* * *$ \\
\hline Year-fixed effect & Yes & & Yes & & Yes & \\
\hline Industry-fixed effect & Yes & & Yes & & Yes & \\
\hline [F-value] & {$[17.08]$} & $* * *$ & {$[17.08]$} & $* * *$ & [16.65] & $* * *$ \\
\hline$R^{2}$ & 0.093 & & 0.093 & & 0.132 & \\
\hline $\mathrm{N}$ & 7533 & & 7533 & & 7533 & \\
\hline
\end{tabular}

Notes: Table 7 reports the results from 2SLS regressions to test the effect of analysts' group affiliation on labor investment efficiency. Panel A presents the results of the first stage, where analysts' group affiliation variables (GAGF, GANGF, NGAGF, NGANGF) are instrumented with five variables (MKV, ROA, GROWTH, EXFINACT, CFVolatility). Panel B presents the estimates from the second-stage regressions. The dependent variables are the absolute value of abnormal net hiring (|AB_NET_HIRE|) for the full sample (Column (1)), and a signed abnormal net hiring ( $\left.A B \_N E T \_H I R E\right)$ for subsample analyses (Columns (2) and (3)). T-statistics are calculated based on robust standard errors clustered at the firm level and are reported in parentheses below the coefficient estimates. The F-value is reported in square brackets. Statistical significance at the 1,5 , and $10 \%$ levels is denoted by ${ }^{* * *}, * *$, and *, respectively. See Appendix A for variable definitions.

\subsection{Sensitivity Test-Matching Firm Size}

Lastly, as a robustness test, we use size matching to reduce potential measurement error in the main regression model. In particular, we divided firms into two groups, one with firms that have a positive number of group analysts following nongroup or unaffiliated firms (GANGF) and another with firms that have a zero GANGF value. Based on firm size, the top and bottom $10 \%$ of the treatment sample (positive GANGF group) is matched with a control sample (zero GAGNGF group) using a random selection method, which is similar to what Mo and Lee suggested [23].

Table 8 shows the results after using size matching. As shown in the table, after we match the treatment sample to the control group by size, the coefficients of analyst following become insignificant in the full sample (column (1)). With an overinvestment subsample, there is a negative association between nongroup analysts following and labor investment efficiency of group firms, indicating that nongroup analysts negatively affect group firms' overinvestment in labor. However, when it comes to the underinvestment problem, both affiliated and unaffiliated analysts enhance group firms' labor investment efficiency. In sum, the results suggest that analyst following generally helps group firms overcome labor underinvestment, but do not help them overcome overinvestment (Table 8). 
Table 8. Size matching.

\begin{tabular}{|c|c|c|c|c|c|c|}
\hline & \multicolumn{6}{|c|}{ Dependent Variable: $\left|A B \_N E T \_H I R E\right|$} \\
\hline & \multicolumn{2}{|c|}{ Full Sample } & \multicolumn{2}{|c|}{$A B \_N E T \_H I R E>0$} & \multicolumn{2}{|c|}{$A B \_N E T \_H I R E<0$} \\
\hline Independent Variables & (1) & & & & & \\
\hline Intercept & $\begin{array}{l}-0.0595 \\
(-0.54)\end{array}$ & & $\begin{array}{l}0.4423 \\
(2.39)\end{array}$ & $* *$ & $\begin{array}{c}-0.0622 \\
(-0.62)\end{array}$ & \\
\hline GAGF & $\begin{array}{l}-0.0655 \\
(-1.14)\end{array}$ & & - & & $\begin{array}{c}-0.0862 \\
(-1.90)\end{array}$ & * \\
\hline GANGF & $\begin{array}{l}0.0154 \\
(1.51)\end{array}$ & & $\begin{array}{l}0.0202 \\
(1.22)\end{array}$ & & $\begin{array}{c}-0.0051 \\
(-0.57)\end{array}$ & \\
\hline NGAGF & $\begin{array}{l}0.0134 \\
(0.72)\end{array}$ & & $\begin{array}{l}0.0767 \\
(1.88)\end{array}$ & * & $\begin{array}{c}-0.0093 \\
(-1.77)\end{array}$ & * \\
\hline NGANGF & $\begin{array}{l}-0.0043 \\
(-1.14)\end{array}$ & & $\begin{array}{l}-0.0103 \\
(-1.62)\end{array}$ & & $\begin{array}{l}0.0015 \\
(0.30)\end{array}$ & \\
\hline MTB & $\begin{array}{l}6.3553 \\
(1.37)\end{array}$ & & $\begin{array}{l}9.0011 \\
(1.02)\end{array}$ & & $\begin{array}{l}1.2786 \\
(0.23)\end{array}$ & \\
\hline SIZE & $\begin{array}{l}0.0044 \\
(0.55)\end{array}$ & & $\begin{array}{c}-0.0070 \\
(-0.44)\end{array}$ & & $\begin{array}{l}0.0144 \\
(1.92)\end{array}$ & * \\
\hline Quick & $\begin{array}{l}0.0004 \\
(0.07)\end{array}$ & & $\begin{array}{c}-0.0072 \\
(-0.52)\end{array}$ & & $\begin{array}{c}0.0072 \\
(1.46)\end{array}$ & \\
\hline$L E V$ & $\begin{array}{l}-0.0308 \\
(-0.28)\end{array}$ & & $\begin{array}{l}-0.0381 \\
(-0.20)\end{array}$ & & $\begin{array}{l}0.0243 \\
(0.15)\end{array}$ & \\
\hline DIVDUM & $\begin{array}{l}-0.0262 \\
(-1.57)\end{array}$ & & $\begin{array}{c}0.0114 \\
(0.41)\end{array}$ & & $\begin{array}{c}-0.0563 \\
(-2.88)\end{array}$ & $* * *$ \\
\hline STD_CFO & $\begin{array}{l}0.0000 \\
(0.27)\end{array}$ & & $\begin{array}{c}0.0000 \\
(0.19)\end{array}$ & & $\begin{array}{l}0.0000 \\
(0.14)\end{array}$ & \\
\hline STD_SALE & $\begin{array}{l}0.0000 \\
(-1.39)\end{array}$ & & $\begin{array}{l}0.0000 \\
(-1.07)\end{array}$ & & $\begin{array}{c}0.0000 \\
(0.59)\end{array}$ & \\
\hline TANGIBLE & $\begin{array}{l}-0.0988 \\
(-2.65)\end{array}$ & $* * *$ & $\begin{array}{c}-0.1287 \\
(-1.93)\end{array}$ & * & $\begin{array}{c}-0.0597 \\
(-1.67)\end{array}$ & * \\
\hline LOSS & $\begin{array}{l}0.0472 \\
(2.30)\end{array}$ & $* *$ & $\begin{array}{c}0.0430 \\
(1.21)\end{array}$ & & $\begin{array}{l}0.0398 \\
(2.01)\end{array}$ & $* *$ \\
\hline INSTI & $\begin{array}{l}-0.0909 \\
(-2.70)\end{array}$ & $* * *$ & $\begin{array}{c}-0.1063 \\
(-1.54)\end{array}$ & & $\begin{array}{c}-0.1185 \\
(-2.10)\end{array}$ & $* *$ \\
\hline STD_NET_HIRE & $\begin{array}{l}0.0557 \\
(2.93)\end{array}$ & $* * *$ & $\begin{array}{l}0.1058 \\
(3.05)\end{array}$ & $* * *$ & $\begin{array}{l}0.0231 \\
(1.05)\end{array}$ & \\
\hline Year-fixed effect & Yes & & Yes & & Yes & \\
\hline Industry-fixed effect & Yes & & Yes & & Yes & \\
\hline [F-value] & [59.08] & $* * *$ & [79.99] & $* * *$ & [4.72] & $* * *$ \\
\hline$R^{2}$ & 0.186 & & 0.321 & & 0.311 & \\
\hline $\mathrm{N}$ & 1710 & & 1054 & & 656 & \\
\hline
\end{tabular}

Notes: Table 8 reports the results of estimating Model (2) after matching firms by their sizes. The dependent variables are an absolute value of abnormal net hiring (|AB_NET_HIRE|) for the full sample (Column (1)), and a signed abnormal net hiring ( $\left.A B \_N E T \_H I R E\right)$ for subsample analyses (Columns (2) and (3)). The independent variable of interest is one of the analysts' group affiliation variables (GAGF, GANGF, NGAGF, NGANGF). T-statistics are calculated based on robust standard errors clustered at the firm level and are reported in parentheses below the coefficient estimates. The F-value is reported in square brackets. Statistical significance at the 1,5, and $10 \%$ levels is denoted by ${ }^{* * *}, * *$, and *, respectively. See Appendix A for variable definitions.

\section{Conclusions}

Despite the importance of analysts' independence, it has not been sufficiently emphasized as one of the virtues of analysts. Prior studies have shown that affiliated analysts are more likely to issue inaccurate earnings forecasts and biased recommendations. This implies that analysts' group affiliation lowers their independence, thus decreasing the quality of information they provide to the market. 
In this paper, we examined whether analysts' group affiliation affects corporate decisions, which go beyond earnings forecasts and stock recommendations.

We studied the relationship between analysts' group affiliation and firms' labor investment decisions. Analysts respond to firms' use of strategic staff planning, and they advance a view on corporate employment decisions. Considering that companies listen to what analysts say, it was expected that analyst following would influence the efficiency of firms' employment decisions. In this paper, we found that there is an increase in the labor investment efficiency of group firms when unaffiliated analysts following increases. The results from further tests showed that an increase in the efficiency of labor investment is driven by resolving the underinvestment problem, especially in decreasing firms' over-firing problem. On the other hand, when analysts are affiliated with firms they follow, their coverage does not affect firms' labor investment efficiency. Additional analyses prove that the positive association between unaffiliated analyst coverage and labor investment efficiency becomes stronger when there is high cashflow within firms. Our results are robust to different model specifications as well.

The relationship between analysts and corporate decisions is well examined in the earlier literature: researchers found that corporate decisions affect both analysts' coverage and the quality of their forecasts and stock recommendations, while other researchers also found that analysts affect various corporate decisions by the firms they follow [10]. Although this paper focuses on analysts' role in firms' employment investment decisions, future research can examine how analysts' independency improves the efficiency of other types of corporate decisions, such as merge and acquisitions, firm restructuring, and investments in IT system.

Author Contributions: Conceptualization and methodology, K.M.; writing original draft and review, K.Y.L.

Funding: This work was supported by the Ministry of Education of the Republic of Korea and the National Research Foundation of Korea (NRF-2019S1A5A8032207).

Conflicts of Interest: The authors declare no conflict of interest.

\section{Appendix A}

Table A1. Variable Definitions.

\begin{tabular}{|c|c|}
\hline Variable Name & Variable Definition \\
\hline \multicolumn{2}{|l|}{ Dependent Variables } \\
\hline NET_HIRE & The percentage change in the number of employees \\
\hline AB_NET_HIRE & 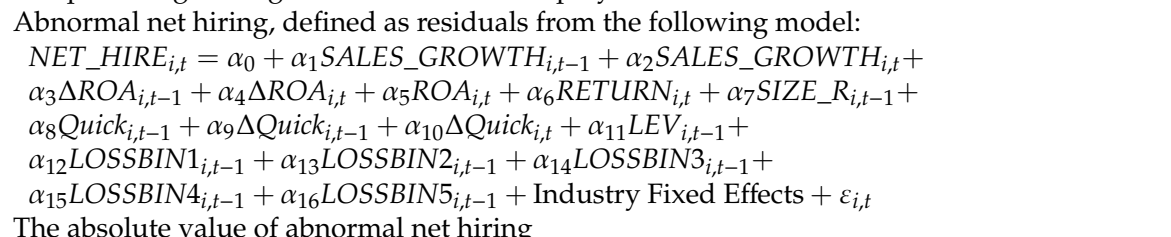 \\
\hline \multicolumn{2}{|c|}{ Analyst Following Variables } \\
\hline Analyst Following & The number of analysts covering a firm \\
\hline GAGF & The number of affiliated analysts following within-group firms \\
\hline GANGF & The number of affiliated analysts following unaffiliated firms \\
\hline NGAGF & The number of unaffiliated analysts following group firms \\
\hline NGANGF & The number of unaffiliated analysts following nongroup firms \\
\hline residual(GAGF) & $\begin{array}{l}\text { The residual coverage of affiliated analysts on within-group firms, defined as residuals from } \\
\text { the following model: } \\
G A G F_{i, t}=\alpha_{0}+\beta_{1} M K V_{i, t-1}+\beta_{2} R O A_{i, t-1}+\beta_{3} G R O W T H_{i, t-1}+\beta_{4} E X F I N A C T_{i, t-1}+ \\
\gamma_{1} C F \text { Volatility } y_{i, t-1}+\text { Year Fixed Effects }+\varepsilon_{i, t}\end{array}$ \\
\hline residual(GANGF) & $\begin{array}{l}\text { The residual coverage of affiliated analysts on unaffiliated firms, defined as residuals from the } \\
\text { following model: } \\
\text { GANGF }_{i, t}=\alpha_{0}+\beta_{1} M K V_{i, t-1}+\beta_{2} R O A_{i, t-1}+\beta_{3} G R O W T H_{i, t-1}+\beta_{4} E_{\text {XXINACT }} T_{i, t-1}+ \\
\gamma_{1} \text { CFVolatility } y_{i, t-1}+\text { Year Fixed Effects }+\varepsilon_{i, t}\end{array}$ \\
\hline
\end{tabular}


Table A1. Cont.

\begin{tabular}{|c|c|}
\hline Variable Name & Variable Definition \\
\hline \multicolumn{2}{|c|}{ Analyst Following Variables } \\
\hline residual(NGANGF) & $\begin{array}{l}\text { The residual coverage of unaffiliated analysts on group firms, defined as residuals from the } \\
\text { following model: } \\
N G A G F_{i, t}=\alpha_{0}+\beta_{1} M K V_{i, t-1}+\beta_{2} R O A_{i, t-1}+\beta_{3} G R O W T H_{i, t-1}+\beta_{4} E X F I N A C T_{i, t-1}+ \\
\gamma_{1} C F V \text { olatility } y_{i, t-1}+\text { Year Fixed Effects }+\varepsilon_{i, t} \\
\text { The residual coverage of unaffiliated analysts on nongroup firms, defined as residuals from the } \\
\text { following model: } \\
\text { NGANGF }{ }_{i, t}=\alpha_{0}+\beta_{1} M K V_{i, t-1}+\beta_{2} R O A_{i, t-1}+\beta_{3} G R O W T H_{i, t-1}+\beta_{4} E X F I N A C T_{i, t-1}+ \\
\gamma_{1} C F V \text { latility } y_{i, t-1}+\text { Year Fixed Effects }+\varepsilon_{i, t}\end{array}$ \\
\hline \multicolumn{2}{|c|}{ Control Variables of Regression Model (1) } \\
\hline SALES_GROWTH & The percentage change in sales revenue \\
\hline$R O A$ & Return on assets, calculated by net income divided by the beginning balance of total assets \\
\hline$\triangle R O A$ & The change in ROA \\
\hline RETURN & Total annual stock returns \\
\hline SIZE_R & The natural logarithm of the market value of equity at the beginning of the year \\
\hline Quick & $\begin{array}{l}\text { Quick ratio, calculated by the sum of cash and cash equivalents, short-term investments, } \\
\text { and receivables divided by current liabilities }\end{array}$ \\
\hline$\Delta$ Quick & The change in the quick ratio \\
\hline LEV & Debt ratio, calculated by long-term liabilities divided by the beginning balance of total assets \\
\hline LOSSBIN1 & $\begin{array}{l}\text { Indicator variable that equals one if a firm's ROA in the prior year is in between }-0.005 \text { and } 0 \text {, } \\
\text { and zero otherwise }\end{array}$ \\
\hline LOSSBIN2 & $\begin{array}{l}\text { Indicator variable that equals one if a firm's ROA in the prior year is in between }-0.01 \text { and } \\
-0.005 \text {, and zero otherwise }\end{array}$ \\
\hline LOSSBIN3 & $\begin{array}{l}\text { Indicator variable that equals one if a firm's ROA in the prior year is in between }-0.015 \text { and } \\
-0.01 \text {, and zero otherwise }\end{array}$ \\
\hline LOSSBIN4 & $\begin{array}{l}\text { Indicator variable that equals one if a firm's ROA in the prior year is in between }-0.02 \text { and } \\
-0.015 \text {, and zero otherwise }\end{array}$ \\
\hline LOSSBIN5 & $\begin{array}{l}\text { Indicator variable that equals one if a firm's ROA in the prior year is in between }-0.025 \text { and } \\
-0.02 \text {, and zero otherwise }\end{array}$ \\
\hline \multicolumn{2}{|c|}{ Control Variables of Regression Model (2) } \\
\hline MTB & Market-to-book ratio, calculated from market value of equity divided by book value of equity \\
\hline SIZE & The natural $\log$ of the market value of equity. \\
\hline DIVDUM & Indicator variable that equals one if a firm pays a dividend, and zero otherwise \\
\hline STD_CFO & The standard deviation of cash flows from operations over the recent 5 years \\
\hline STD_SALE & The standard deviation of sales revenue from operations over the recent 5 years \\
\hline TANGIBLE & The ratio of long-term assets to the beginning balance of total assets \\
\hline LOSS & Indicator variable that equals one if a firm has a net loss, and zero otherwise \\
\hline INSTI & The percentage of shares owned by institutional investors \\
\hline STD_NET_HIRE & The standard deviation of net hiring \\
\hline \multicolumn{2}{|c|}{ Variables Used in Additional Tests } \\
\hline High (Low) Fund & $\begin{array}{l}\text { A firm is considered to have a high (low) level of inside fund when its cash and cash } \\
\text { equivalents scaled by total assets is above (below) the yearly median }\end{array}$ \\
\hline MKV & Market value \\
\hline GROWTH & The growth rate of total assets \\
\hline EXFINACT & Net cash proceeds from external financing scaled by total assets \\
\hline CFVolatility & The standard deviation of cash flow scaled by last year's total assets \\
\hline
\end{tabular}

\section{References}

1. Brown, L.D.; Call, A.C.; Clement, M.B.; Sharp, N.Y. Inside the "black box" of sell-side financial analysts. J. Account. Res. 2015, 53, 1-47. [CrossRef]

2. Graham, J.R.; Harvey, C.R.; Rajgopal, S. The economic implications of corporate financial reporting. J. Account. Econ. 2005, 40, 3-73. [CrossRef]

3. Yu, F.F. Analyst coverage and earnings management. J. Financ. Econ. 2008, 88, 245-271. [CrossRef]

4. Chen, T.; Harford, J.; Lin, C. Do analysts matter for governance? Evidence from natural experiments. J. Financ. Econ. 2015, 115, 383-410. [CrossRef]

5. Keitz, A.; Terrarosa, T. Ford CEO Jim Hackett's Restructuring Plans Provoke Skepticism as Shares Slide. Available online: https:/www.thestreet.com/investing/ford-restructuring-plans-receives-mixed-reviews14663958 (accessed on 29 May 2019). 
6. Hiller, J. Occidental to Sell Parts of Anadarko after Debt-Fueled Acquisition. Available online: https://www.reuters.com/article/us-occidental-divestitures/occidental-to-sell-parts-of-anadarkoafter-debt-fueled-acquisition-idUSKCN1SY0XW (accessed on 29 May 2019).

7. Wells, J. Retail Labor Cuts are Hurting In-Store Experience, Analysts Say. Available online: https://www.grocerydive.com/news/grocery--retail-labor-cuts-are-hurting-in-store-experienceanalysts-say/535045/ (accessed on 2 May 2019).

8. Finkle, J. Cisco Braces for Biggest Layoffs in Its History. Available online: https://www.reuters.com/article/uscisco/cisco-braces-for-biggest-layoffs-in-its-history-idUSTRE74A78K20110513 (accessed on 2 May 2019).

9. Xu, J.; Sim, J.W. Characteristics of corporate R\&D investment in emerging markets: Evidence from manufacturing industry in China and South Korea. Sustainability 2018, 10, 3002.

10. Oh, H.; Kim, W. The effect of analyst coverage on the relationship between seasoned equity offerings and investment efficiency from Korea. Sustainability 2018, 10, 2704. [CrossRef]

11. Huyghebaert, N.; Xu, W. Bias in the post-IPO earnings forecasts of affiliated analysts: Evidence from a Chinese natural experiment. J. Account. Econ. 2016, 61, 486-505. [CrossRef]

12. Pae, P. South Korea's Chaebol. Available online: https://www.washingtonpost.com/business/southkoreas-chaebol/2018/10/05/e27095ac-c871-11e8-9c0f-2ffaf6d422aa_story.html?noredirect=on\&utm_term= .872e52026fe3 (accessed on 2 May 2019).

13. Lim, Y.; Jung, K. Conflict of interest or information sharing? Evidence from affiliated analyst performance in Korea. Contemp. Account. Res. 2012, 29, 505-537. [CrossRef]

14. Lee, C. A Company that Is 'Jumping' to An Analyst. Available online: https://translate.google.com/translate? hl=en\&sl=ko\&u=http://biz.chosun.com/site/data/html_dir/2016/04/18/2016041802246.html\&prev=search (accessed on 2 May 2019).

15. Kwak, B.; Mo, K. Group-affiliated analysts' strategic forecasts during a year: Evidence from Korea. Emerg. Mark. Financ. Trade 2019, 55, 59-77. [CrossRef]

16. Mantecon, T.; Altintig, Z.A. Chaebol-affiliated analysts: Conflicts of interest and market responses. J. Bank. Financ. 2012, 36, 584-596.

17. Jung, B.; Lee, W.J.; Weber, D.P. Financial reporting quality and labor investment efficiency. Contemp. Account. Res. 2014, 31, 1047-1076. [CrossRef]

18. Pinnuck, M.; Lillis, A.M. Profits versus losses: Does reporting an accounting loss act as a heuristic trigger to exercise the abandonment option and divest employees? Account. Rev. 2007, 82, 1031-1053. [CrossRef]

19. Biddle, G.C.; Hilary, G.; Verdi, R.S. How does financial reporting quality relate to investment efficiency? J. Account. Econ. 2009, 48, 112-131. [CrossRef]

20. Biddle, G.C.; Hilary, G. Accounting quality and firm-level capital investment. Account. Rev. 2006, 81, 963-982. [CrossRef]

21. Liu, M.; Wysocki, P. Cross-sectional determinants of information quality proxies and cost of capital measures. Q. J. Financ. 2017, 7, 1650016. [CrossRef]

22. Cella, C. Institutional investors and corporate investment. Financ. Res. Lett. 2019. [CrossRef]

23. Mo, K.; Lee, J. IFRS adoption and the choice between public and private debt: Evidence from South Korea. Emerg. Mark. Financ. Trade 2018, 54, 2533-2556. [CrossRef]

(C) 2019 by the authors. Licensee MDPI, Basel, Switzerland. This article is an open access article distributed under the terms and conditions of the Creative Commons Attribution (CC BY) license (http://creativecommons.org/licenses/by/4.0/). 